\title{
Stimulated radar collider for probing gravitationally weak coupling pseudo Nambu-Goldstone bosons
}

\author{
Kensuke Homma ${ }^{a, b, 1}$ and Yuri Kirita ${ }^{b}$ \\ ${ }^{a}$ Graduate School of Advanced Science and Engineering, Hiroshima University, \\ 1-3-1 Kagamiyama, Higashi-Hiroshima 739-8526, Japan \\ ${ }^{b}$ Graduate School of Science, Hiroshima University, \\ 1-3-1 Kagamiyama, Higashi-Hiroshima 739-8526, Japan \\ E-mail: khomma@hiroshima-u.ac.jp, kirita@quark.hiroshima-u.ac.jp
}

\begin{abstract}
We propose a stimulated pulsed-radar collider to directly produce pseudo Nambu-Goldstone bosons as candidates for dark components in the Universe and simultaneously induce the decay by mixing two radar beams. We have extended formulae for stimulated resonant photon-photon scattering in a quasi-parallel collision system by including fully asymmetric collision cases. With a pulse energy of $100 \mathrm{~J}$ in the GHz-band, for instance, which is already achieved by an existing klystron, we expect that the modelindependent sensitivity can reach gravitationally weak coupling domains at a mass range $10^{-7}-10^{-6} \mathrm{eV}$, if two key technological issues are resolved: pulse compression in time reaching the Fourier transform limit, and single-photon counting for GHz-band photons. Such testing might extend the present horizon of particle physics.
\end{abstract}

KEYwORDs: Dark matter, Other experiments, Photon production, Exotics

ArXIV EPRINT: 1909.00983

\footnotetext{
${ }^{1}$ Corresponding author.
} 


\section{Contents}

1 Introduction 1

2 Concept of stimulated pulsed-radar collider 4

3 Expected sensitivity 4

4 Conclusion $\quad 8$

A Formulae for the fully asymmetric stimulated resonance photon-photon $\begin{array}{ll}\text { scattering in a quasi-parallel collision system } & \mathbf{9}\end{array}$

A.1 Lorentz-invariant transition amplitude in the sea of coherent fields 9

A.2 Kinematics in asymmetric-incident and non-coaxial geometry in QPS $\quad 14$

$\begin{array}{lll}\text { A.3 Lorentz-invariant scattering amplitude including a resonance state } & 15\end{array}$

$\begin{array}{lll}\text { A.4 Evaluation of signal yield in stimulated resonant scattering } & 17\end{array}$

$\begin{array}{ll}\text { A.4.1 Properties of a Gaussian beam in vacuum } & 19\end{array}$

A.4.2 Integrated inducible volume-wise interaction rate, $\bar{\Sigma}_{I} \quad 21$

A.4.3 Spacetime overlapping factor with an inducing beam, $\mathcal{D}_{I} \quad 23$

\section{Introduction}

Since Rutherford's experiment, the observation of quantum scattering processes caused by colliding energetic charged particles has unveiled deeper layers of nature at the microscopic. With knowledge gleaned from these particle collisions, the Standard Model (SM) of elementary particles is now almost confirmed, with the recent discovery of the Higgs boson providing another point of evidence for the SM. However, the SM is still unsatisfactory when trying to quantitatively understand the profile of the energy density of the universe as evaluated from macroscopic gravitational observables through curvatures in spacetime. In particular, atoms consisting of elements in the SM can explain only $\sim 5 \%$ of the observed energy density of the universe. The remainder of the energy density is assumed to be accounted for by dark matter and dark energy [1]. Why, then, is so little understood about dark components?

The gravitational coupling strength $G_{N} \sim 10^{-38} \mathrm{GeV}^{-2}(\hbar=c=1)$ is extraordinary weak, even relative to the weakest coupling strength of the weak interaction $G_{F} \sim$ $10^{-5} \mathrm{GeV}^{-2}$ among the SM. Because of its extraordinary weakness, gravitational coupling has never been probed by elementary scattering processes. In this sense, gravity has been, in practice, beyond the scope of experimental particle physics to date. Therefore, it is unlikely that present knowledge of particle physics is sufficient to understand dark components obtained from gravitational observables. We suggest in this paper that we can 
actually test scattering processes, even with gravitationally weak coupling, if a properly designed stimulated photon-photon collider is used. Such testing might extend the present horizon of particle physics.

Massless Nambu-Goldstone fields accompany spontaneous breaking of global continuous symmetries $[2,3]$. The neutral pion is a typical Nambu-Goldstone Boson (NGB). However, the physical mass is slightly greater than zero. This pseudo-NGB (pNGB) state is caused by chiral symmetry breaking in quantum chromodynamics (QCD). However, pNGBs are not limited to chiral symmetry. In general, whenever a global symmetry of any type is broken, we may expect a pNGB to exist. This viewpoint can be used as a robust guiding principle to search for something very low in mass in the Universe, even without knowing the details of individual dynamics. If a particle collider could directly produce pNGB resonance states via gravitationally weak interactions, for instance, generic string-inspired scalar fields as discussed in [4] can be directly searched for. The proposed method in this paper would open up such a possibility.

To directly probe low-mass pNGBs, photon-photon scattering has special advantages arising from the coupling of two photons, because photons are massless and the center-ofmass system $(\mathrm{cms})$ energy, $E_{\mathrm{cms}}$, can be extremely low in comparison with that of charged particle collisions. We therefore discuss the following effective Lagrangian, which expresses coupling to two photons:

$$
-\mathcal{L}=g M^{-1} \frac{1}{4} F_{\mu \nu} F^{\mu \nu} \phi
$$

where a scalar-type field $\phi$ with effective coupling $g / M$ to two photons is assumed as an example of pNGB. If $M$ corresponds to the Planckian mass $M_{\mathrm{P}}=\sqrt{c \hbar /\left(8 \pi G_{N}\right)}=$ $2.44 \times 10^{18} \mathrm{GeV}(\hbar=c=1)$, the coupling is as weak as that of gravity.

We have advocated that stimulated photon-photon scattering in a Quasi-Parallel collision System (QPS), illustrated in figure 1a, can drastically enhance the interaction rate [5]. Capturing a resonance state in an $s$-channel photon-photon scattering within the uncertainty on $E_{\text {cms }}$ is the first key element of the proposed method. The second key element is the enhancement of the interaction rate by the technique discussed in appendix, which relies on the stimulated nature of the two-body photon-photon scattering process, adding a coherently co-propagating field as the inducing field. Among several possible collision geometries [5-9], QPS is the optimum geometry for the low mass range, having the widest accessible mass range possible for a single collision geometry. For simplicity, we have initially considered QPS with a symmetric incident angle $\vartheta$, as shown in figure 1a [5]. This can be realized by focusing a photon beam with a single photon energy $\omega$. In this case, $E_{\text {cms }}$ is expressed as

$$
E_{\mathrm{cms}}=2 \omega \sin \vartheta .
$$

This allows experiments to have two knobs to handle $E_{\mathrm{cms}}$. The choice of combination between photon energies and incident angles depends on the trade-off between the beam and sensor technologies. In QPS, description of the interaction is non-trivial due to the inherently wave-like nature of photons [6]. As we show in detail in appendix, the interaction rate is increased when electromagnetic waves are confined to a short time scale. If the waves are confined to a short duration, then an energy uncertainty $\delta \omega$ must be introduced 
(a)

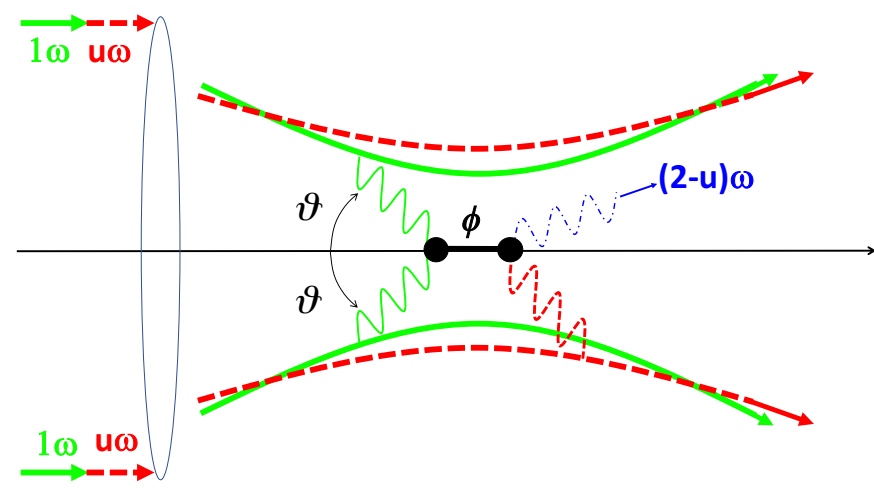

(b)

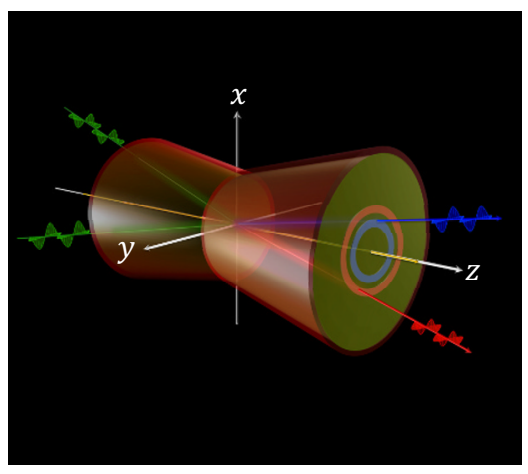

(c)

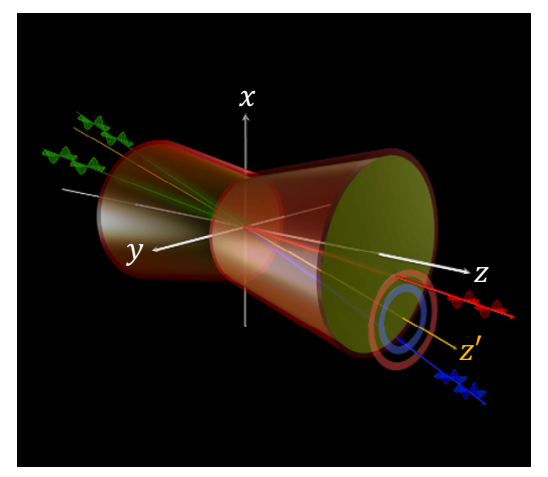

Figure 1. Stimulated resonant scattering in a quasi-parallel collision system (QPS) and the classification of collision geometries. (a) Conceptual drawing of stimulated resonant photon-photon scattering in QPS, realizable when a coherent field with energy $\omega$ (solid green line) is combined with another coherent field with energy $u \omega(0<u<1)$ (dashed red line) and both fields are focused by a lens element in vacuum. The emission of signal photons with energy $(2-u) \omega$ (dash-dotted blue line) is stimulated as a result of energy-momentum conservation in the scattering process $\omega+\omega \rightarrow \phi \rightarrow(2-u) \omega+u \omega$ via a resonance state $\phi$. (b) Symmetric-incident and coaxial scattering, where the incident angles of two photon wave vectors and their energies are symmetric, and the transverse momenta of photon pairs, $p_{T}$, always vanish with respect to the common optical axis $z$. (c) Asymmetric-incident and non-coaxial scattering, where the incident angles of two photon wave vectors and their energies are asymmetric, resulting in a finite value of $p_{T}$ with respect to the common optical axis $z$. The zero- $p_{T}$ axis $\left(z^{\prime}\right.$-axis) is always configurable for arbitrary pairs of two incident wave vectors.

according to the uncertainty principle for the energy-time relation or, equivalently, as a result of the Fourier transform from the time domain to the frequency domain. In addition, around the focal spot, the momentum uncertainty is also maximized due to the spatial localization of a beam field again based on the uncertainty principle for the momentum-space relation. This implies that the incident angles of electromagnetic waves must also fluctuate strongly. These situations require us to depart from the simplest geometry (i.e., from assuming symmetric energies and symmetric incident angles) and use a fully asymmetric geometry in QPS, as illustrated in figure 1c. The extended parametrization associated with the fully asymmetric case is non-trivial, and we will show this in appendix. The main 
finding allowed by the extension to the fully asymmetric case is that the probability that non-coaxial collisions (figure 1c) will occur dominates the probability of coaxial collisions (figure 1b).

In the following sections, we first consider the concept of the stimulated pulsed-radar collider. We then evaluate the expected sensitivity based on the parametrization including the fully asymmetric collision cases in QPS. In order to reach the gravitationally coupling pNGBs, we discuss two technological requirements toward the future laboratory search. Finally, our conclusion is given.

\section{Concept of stimulated pulsed-radar collider}

Toward the direct detection of pNGBs at around a target mass range $m \sim 10^{-7} \mathrm{eV}$, if we consider only a laser source with single-photon energy $\sim 1 \mathrm{eV}$, the incident angle must be maintained at $\sim 10^{-7}$ radians and it is likely impossible to perform the search on the ground with a focal distance greater than $10^{7} \mathrm{~m}$, assuming a beam diameter of $\sim 1 \mathrm{~m}$. However, if we could use an energy of $\omega \sim 10^{-5} \mathrm{eV}$ with incident angle $\vartheta \sim 10^{-2}$ radians, it would be possible to focus the beam to within a few hundred meters for a beam having a diameter of a few meters and wavelength below $30 \mathrm{~cm}$ ( $1 \mathrm{GHz}$ in frequency).

Indeed, intense sources of $\mathrm{GHz}$ photons are already commercially available, such as the klystron [10]. Moreover, the number of photons per pulse is $\sim 10^{5}$ times that of optical laser fields for the same pulse energy. Therefore, pulsed-radar beam in the GHz-band would be useful for a mass domain of $\sim 10^{-7} \mathrm{eV}$. However, as we will discuss in the next section, pulse compression will be demanded in order to reach the gravitationally weak coupling domain. This requires future developments for the pulse compression technique in the $\mathrm{GHz}$-band.

Furthermore, $\mathrm{GHz}$ photon counting with single-photon sensitivity is a difficult technological issue. In the area of GHz-photon sensing, some successful examples of single-photon detection with quantum-bit (qubit) technology have been reported recently [11, 12]. In particular, a phase-qubit sensor based on Josephson junctions [11] can be operated with a pulsed current bias within several ns duration [11]. This time-gated operation would reduce dark currents from continuous background blackbody radiation. Using the above considerations, figure 2 illustrates a conceptual setup for a pNGB search in QPS with GHzphoton sources equipped with a phase-qubit-type GHz-photon counter for the detection of signal photons.

\section{Expected sensitivity}

Given this stimulated radar collider setup with the experimental parameters set as listed in table 1 , we discuss how we can reach the gravitational coupling domain in $g / M<$ $\alpha_{\text {qed }} / M_{\mathrm{P}}$, based on new formulas including an asymmetric quasi-parallel collision geometry, explained in appendix in detail. In symmetric-incident and coaxial scattering in QPS (figure 1b), transverse momenta of photon pairs, $p_{T}$, always vanish with respect to the common optical axis $z$. This guarantees that azimuthal angles of the final state photon 


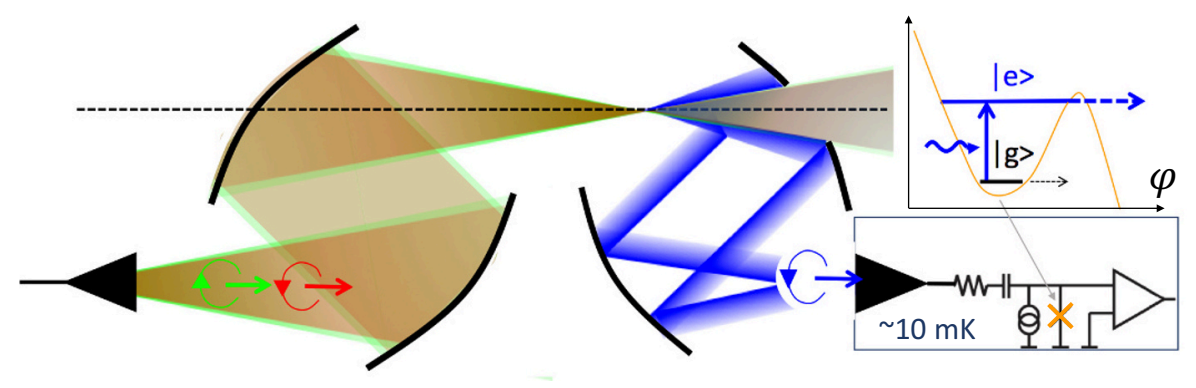

Figure 2. Conceptual setup of the simulated radar collider and the detection of signal photons. Two circularly polarized coherent beams for creation (green, left-handed) and stimulation (red, right-handed) are combined and focused along a common optical axis. Signal photons (blue, righthanded) are emitted via the exchange of a pNGB. Around the focal plane, only signal photons are partially reflected and collimated by a dichroic parabolic mirror with a hole through which intense $\mathrm{GHz}$ beams can escape the detection system, both to avoid adding thermal background sources and to avoid picking up atomic four-wave mixing processes from the upstream mirror surfaces as well as from residual gases in the focal spot, because these background photons are expected to be confined within the incident angles of the two beams. These peripherally emitted signal photons are focused into the detector element. The detector consists of a signal photon counter. For sensing $\mathrm{GHz}$ photons, for instance, a reasonable candidate is a Josephson-junction sensor based on a pulsecurrent-biased phase-qubit [11]. The bias instantaneously forms a potential, illustrated in the inset, as a function of the phase difference $\varphi$ between two superconductors sandwiching an insulator gap. When a GHz-photon is absorbed in one of the two superconductors, the energy state of a Cooper pair transits from the ground state $|g\rangle$ to an excited state $|e\rangle$, which drastically increases the probability for the Cooper pair to tunnel to the neighboring superconductor though the gap. This allows number-resolved counting if parallelized junctions are implemented [11].

wave vectors are axially symmetric around the $z$-axis. Therefore, the inducible momentum or angular range can be analytically obtained via the axial symmetric nature of the focused beams. On the other hand, in asymmetric-incident and non-coaxial scattering (figure 1c), finite transverse momenta are unavoidably introduced. However, a zero- $p_{T}$ axis, defined as the $z^{\prime}$-axis, is always configurable for any arbitrary pair of two incident wave vectors. Therefore, $z^{\prime}$-axis can restore the axial symmetric nature of the azimuthal angles of the final state wave vectors. Despite this, the inducing coherent field is physically mapped to the common optical axis $z$. Therefore, the inducible momentum range changes in a complicated manner that depends on an arbitrarily formed $z^{\prime}$-axis. Hence, numerical integration must be performed to express the number of signal photons per shot $\mathcal{Y}_{c+i}$ in eq. (A.54) by substituting eqs. (A.80) and (A.84), shown in appendix. The number of experimentally observable signal photons $N_{\text {obs }}$ as a function of mass and coupling for the set of experimental parameters $P$ given in table 1 is then expressed as

$$
N_{\mathrm{obs}}=\mathcal{Y}_{c+i}(m, g / M ; P) t_{a} f \epsilon
$$

where the data acquisition time is $t_{a}$, the repetition rate of pulsed beams is $f$, and the overall efficiency is $\epsilon \equiv \epsilon_{d}\left(\tau_{b} / 2 \tau_{i}\right)$ with detection efficiency $\epsilon_{d}$, qubit current-bias time $\tau_{b}$, and inducing pulse duration $\tau_{i}$. By numerically solving this equation, we can obtain 


\begin{tabular}{|c|c|c|}
\hline Creation radar pulse & Klystron & Compress \\
\hline central frequency $\nu_{c}$ & $2.8 \mathrm{GHz}$ & \\
\hline energy per pulse & $100 \mathrm{~J}$ & \\
\hline repetition rate $f$ & $50 \mathrm{~Hz}$ & \\
\hline relative linewidth $\delta \nu_{c} / \nu_{c} \times 100$ & $\pm 10^{-2} \%$ & $\pm 5 \%$ \\
\hline half pulse duration, $\tau_{c}$ & $1 \mu \mathrm{s}$ & $0.568 \mathrm{~ns}$ \\
\hline Inducing radar pulse & Klystron & Compress \\
\hline central frequency $\nu_{i}$ & $1.6 \mathrm{GHz}$ & \\
\hline energy per pulse & $100 \mathrm{~J}$ & \\
\hline repetition rate $f$ & $50 \mathrm{~Hz}$ & \\
\hline relative linewidth $\delta \nu_{i} / \nu_{i} \times 100$ & $\pm 10^{-2} \%$ & $\pm 5 \%$ \\
\hline half pulse duration, $\tau_{i}$ & $1 \mu \mathrm{s}$ & $0.995 \mathrm{~ns}$ \\
\hline Measurement requirements & Klystron & Compress \\
\hline central signal frequency $\nu_{s}$ & $4 \mathrm{GHz}$ & \\
\hline \# of observed signals $N_{\text {obs }}$ & 100 photons & \\
\hline data acquisition time $t_{a}$ & 1 month $\left(2.6 \times 10^{6}\right)$ & \\
\hline detector current bias time $\tau_{b}$ & $1 \mathrm{~ns}$ & \\
\hline detector efficiency $\epsilon_{d}$ & 0.1 & \\
\hline overall efficiency $\epsilon \equiv \epsilon_{d}\left(\tau_{b} / 2 \tau_{i}\right)$ & $5 \times 10^{-5}$ & $5 \times 10^{-2}$ \\
\hline \multicolumn{3}{|l|}{ Geometric parameters in QPS } \\
\hline creation beam diameter & $6.02 \mathrm{~m}$ & \\
\hline inducing beam diameter & $6.10 \mathrm{~m}$ & \\
\hline common focal length & $30 \mathrm{~m}$ (five Rayleigh & length) \\
\hline
\end{tabular}

Table 1. GHz-band pulsed beam parameters similar to the existing klystron [10] and the compressed case reaching the Fourier transform limit $\tau_{j} \times 2 \pi \delta \nu_{j} \geq 1 / 2$ for $j=c$ or $i$.

$g / M$ for the given values of $m$ and $N_{\text {obs. }}$. Dominant background photons are expected from blackbody radiations in the same spectrum width as that of the signal photons, $(1 \pm \sim 0.05) \nu_{s}$. The unavoidable blackbody source is the entrance horn connected to the qubit senor. The total number of background photons is evaluated as $N_{\mathrm{bkg}}=0.5$ photons by assuming that the horn and sensor temperatures are kept at $T=10 \mathrm{mK}$ with the inner surface area of the cone-type horn $\Delta S=\pi \lambda_{s}^{2}$ for signal photon wavelength $\lambda_{s}=c / \nu_{s}$ and solid angle $\Delta \Omega=2 \pi$ from the following relation

$$
N_{\mathrm{bkg}}=\int_{0.95 \nu_{s}}^{1.05 \nu_{s}} \frac{2 h \nu^{3}}{c^{2}} \frac{1}{e^{h \nu /(k T)}-1} d \nu \frac{\Delta \Omega \Delta S t_{a} f \epsilon}{h \nu_{s}} .
$$

We also note that photon-photon scattering in the SM can be neglected because the QEDbased stimulated scattering is sufficiently suppressed by the $E_{\text {cms }}^{6}$ dependence of the cross section $[9,13]$. Considering systematic backgrounds, we require $N_{\text {obs }}=100 \gg N_{\text {bkg }}$ in this paper.

Figure 3a summarizes the accessible domains for coupling $g / M$ versus mass $m$. Excluded domains (5th force and EP test) and projected sensitivities (quartz and $\mathrm{Cu}-\mathrm{Si}$ 


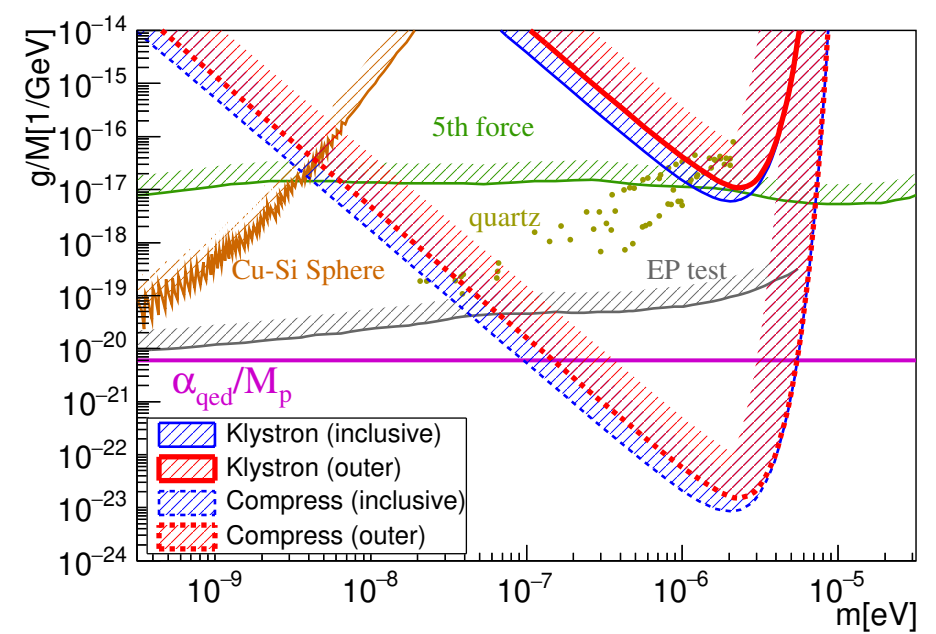

(a)

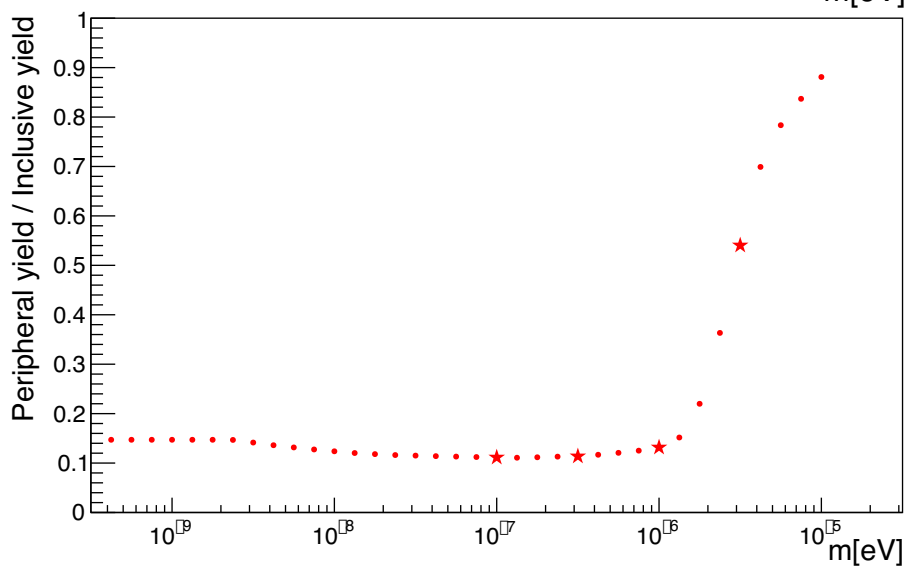

(b)

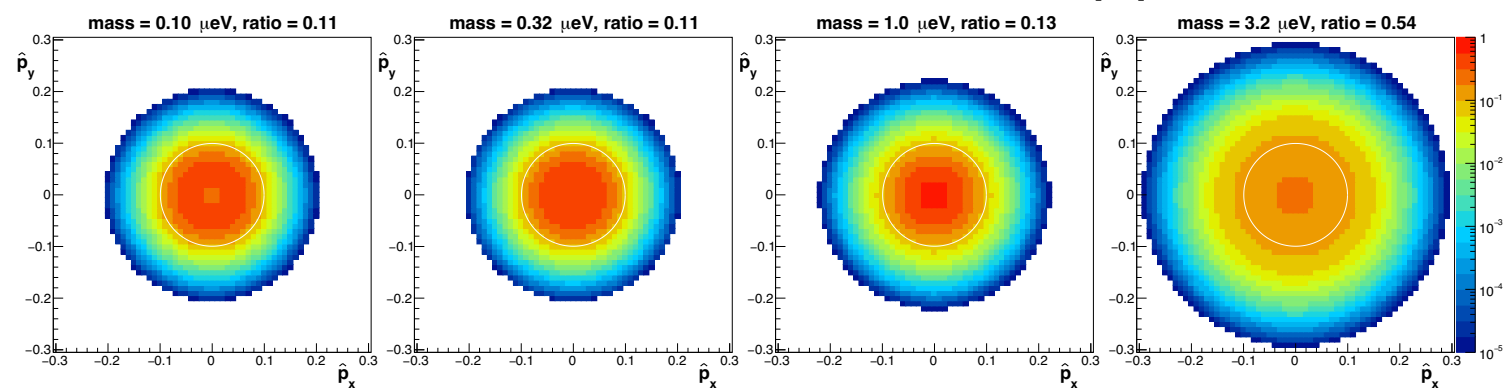

Figure 3. Sensitivity curves and the ratio of outer emissions with the parameters listed in table 1. (a) Reachable coupling $g / M$ vs. pNGB mass $m$ domains. The domains above the solid curves show the reachable range with peak power and relative linewidth of GHz-photon pulses equivalent to an existing $100 \mathrm{MW}$ klystron [10] (see the left column of table 1 for the linewidth and pulse duration parameters). The dotted curves show the extended sensitivities when a relatively broader linewidth (the right column for the same parameters in table 1) is used; it is assumed a system capable of this will be developed in the near future. The thicker curves show the sensitivities when the signal photons are sampled only outside the divergence angles of the incident two beams. Excluded domains (5th force and EP test) and projected sensitivities (quartz and $\mathrm{Cu}-\mathrm{Si}$ Sphere) by the different methods are also superimposed by quoting limits from figure 1 in [4], where the details of individual curves and the relevant references can be found. (b) The ratio of the signal photon yield emitted outside the divergence angles of incident beams to that of the inclusive yield as a function of mass. The lower four panels show the numerically calculated signal yields sampled at four mass points indicated with the star markers in the ratio plot as a function of the $x$ and $y$ components of unit momenta of signal photons, $\left(\hat{p}_{x}, \hat{p}_{y}\right)$, at the beam waist $z=0$; the white circles indicate the domains of beam divergence with the radius of $\sin \Theta_{0}$ from eq. (A.58) in appendix. The color contours in the log scale are normalized to the common total number of signal photons. 
Sphere) by the different methods are also superimposed by quoting limits from figure 1 in [4], where the details of individual curves and the relevant references can be found. This indicates that broadening the linewidth is indeed a key factor because it can increase the interaction rate by increasing the spacetime overlapping factor of the incident pulsed beams, as explained in eq. (A.84), due to the short durations $\tau_{c}$ and $\tau_{i}$, and also increases the chance to stimulate emission of final state photons satisfying energy-momentum conservation within the allowed energy-momentum fluctuations of collision beams, as indicated in eq. (A.80) in appendix. Technologically, however, broadening the linewidth, equivalently, compressing a $\mathrm{GHz}$ pulse below a few ns is a nontrivial issue. In the current particle accelerator technologies, the pulse compression of radio frequency (RF) can be performed based on resonant cavities and transmissionlines. For instance, a double-stage cavity pulse compressor is expected to be able to achieve a final pulse length of $10 \mathrm{~ns}$ but with a significant inefficiency [14]. Another example is the compression of frequency-modulated pulses using helically corrugated waveguides for generating a few ns multi-gigawatt RF pulses [15]. Applying these compression schemes to a 100 gigawatt scale would require further elaborations in order to avoid RF breakdown at such high power. Since eventually the proposed scheme requires collecting and focusing waves by parabolic mirrors in free space, the concept of phased array antenna [16] by coherently combining waves with a sufficiently large area from the individual RF emitters could naturally fit to the proposed setup for preventing RF breakdown as well as forming and directing beams.

Figure $3 \mathrm{~b}$ shows the ratio of signal photons found outside the angular divergence of the focused beams based on the geometric optics as a function of mass. For larger masses, larger fractions of signal photons are emitted to the outer angles. The non-coaxial collisions allow signal photon emission outside the divergence angles of the focused beams defined by geometric optics. Thanks to this scattering behavior, we can expect that the ratio between the number of signal photons and beam photons could be improved if we could measure only peripheral emissions around the common optical axes, as illustrated in figure 2 .

\section{Conclusion}

We have formulated stimulated resonant photon-photon scattering in QPS including asymmetric-incident and non-coaxial collisions. From the stimulated pulsed-radar collider concept, we expect that the sensitivity can reach a weaker coupling domain than the gravitational coupling strength at the mass range $1 \times 10^{-7} \sim 5 \times 10^{-6} \mathrm{eV}$ assuming two key technological issues are resolved: pulse compression in time reaching the Fourier transform limit, and single-photon counting for GHz-band photons. These are possible in principle but technologically challenging in practice. It is worth being striving for them, however, because they would allow direct probing of gravitationally weak scattering processes in laboratory experiments, which has not been done in the history of science.

\section{Acknowledgments}

We express deep gratitude to Yasunori Fujii, who passed away in July 2019. This study is initially motivated by his outstanding works on the dilaton model. We also thank 
K. Ishikawa for discussions about evaluation of the inducing effect, S. Mima and S. Shibata for discussions on the qubit application, and M. Oxborrow for discussions on pulsed $\mathrm{GHz}$ sources. K. Homma acknowledges the support of the Collaborative Research Program of the Institute for Chemical Research, Kyoto University (Grants Nos. 2018-83 and 2019-72) and Grants-in-Aid for Scientific Research Nos. 17H02897, 18H04354, and 19K21880 from the Ministry of Education, Culture, Sports, Science and Technology (MEXT) of Japan.

\section{A Formulae for the fully asymmetric stimulated resonance photon-pho- ton scattering in a quasi-parallel collision system}

Here, we provide full details of the evaluation of signal yield in stimulated resonant photonphoton scattering in a quasi-parallel collision system (QPS) that includes fully asymmetric collision and stimulation geometries due to uncertainties regarding energy and incident angles in QPS. Figure 3 is calculated from numerical integration of eq. (A.54) with eqs. (A.80) and (A.84), using the parameter values given in table 1 . We use the metric convention $(+---)$ throughout this appendix.

\section{A.1 Lorentz-invariant transition amplitude in the sea of coherent fields}

The S-matrix for the interaction Lagrangian

$$
-\mathcal{L}=g M^{-1} \frac{1}{4} F_{\mu \nu} F^{\mu \nu} \phi
$$

is expressed as

$$
S^{(2)}=\left(-\frac{1}{4} \frac{g}{M}\right)^{2} \frac{i^{2}}{2} \int d^{4} x \int d^{4} y T\left[F_{\mu \nu}(x) F^{\mu \nu} \phi(x) F_{\rho \sigma}(y) F^{\rho \sigma}(y) \phi(y)\right],
$$

where $T$ denotes the time-ordered product. From Wick's theorem, the T-product can be converted to the normal-ordering product by requiring contractions with four external electromagnetic fields, as follows.

$$
N\left[F_{\mu \nu}(x) F^{\mu \nu}(x) F_{\sigma \rho}(y) F^{\sigma \rho}(y)\langle 0|T[\phi(x) \phi(y)]| 0\rangle\right] .
$$

Here,

$$
i\langle 0|T[\phi(x) \phi(y)]| 0\rangle \equiv \frac{1}{(2 \pi)^{4}} \int d^{4} q \frac{e^{-i q(x-y)}}{m^{2}-q^{2}-i \epsilon}
$$

is the propagator of a massive scalar field $\phi$ with an infinitesimal number $\epsilon$. We expand the field strength tensor as

$$
F^{\mu \nu} \equiv(-i) \int \frac{d^{3} \boldsymbol{p}}{(2 \pi)^{3} 2 p^{0}} \Sigma_{\lambda=1,2}\left(P^{\mu \nu} e^{-i p x} a_{\boldsymbol{p}, \lambda}+\hat{P}^{\mu \nu} e^{i p x} a_{\boldsymbol{p}, \lambda}^{\dagger}\right)
$$

and further define the following momentum-polarization tensors as capitalized symbols for an arbitrary four-momentum $p$ of the electromagnetic field with the polarization state $\lambda$ :

$$
\begin{aligned}
& P^{\mu \nu} \equiv p^{\mu} \epsilon^{\nu}(p, \lambda)-\epsilon^{\mu}(p, \lambda) p^{\nu}, \\
& \hat{P}^{\mu \nu} \equiv \epsilon^{* \mu}(p, \lambda) p^{\nu}-p^{\mu} \epsilon^{* \nu}(p, \lambda) .
\end{aligned}
$$


The commutation relations are

$$
\begin{aligned}
& {\left[a_{\boldsymbol{k}, \lambda}, a_{\boldsymbol{k}^{\prime}, \lambda^{\prime}}^{\dagger}\right]=(2 \pi)^{3} 2 p^{0} \delta^{3}\left(\boldsymbol{k}-\boldsymbol{k}^{\prime}\right) \delta\left(\lambda-\lambda^{\prime}\right),} \\
& {\left[a_{\boldsymbol{k}, \lambda}, a_{\boldsymbol{k}^{\prime}, \lambda^{\prime}}\right]=\left[a_{\boldsymbol{k}, \lambda}^{\dagger}, a_{\boldsymbol{k}^{\prime}, \lambda^{\prime}}^{\dagger}\right]=0 .}
\end{aligned}
$$

From here, we omit the polarization index $\lambda$ and the sum over it for the photon creation and annihilation operators, $a_{\boldsymbol{p}, \lambda}$ and $a_{\boldsymbol{p}, \lambda}^{\dagger}$, because we require fixed beam polarizations in the last step of the following calculations. Substituting eqs. (A.3)-(A.6) into (A.2), we get

$$
\begin{aligned}
S^{(2)}= & \left(-\frac{1}{4} \frac{g}{M}\right)^{2} \frac{i}{2} \int d^{4} x \int d^{4} y \int \frac{d^{4} q}{(2 \pi)^{4}} \frac{e^{-i q(x-y)}}{m^{2}-q^{2}-i \epsilon} \times \\
& (-i)^{4} \int \frac{d^{3} \boldsymbol{s}}{(2 \pi)^{3} 2 s^{0}} \int \frac{d^{3} \boldsymbol{t}}{(2 \pi)^{3} 2 t^{0}} \int \frac{d^{3} \boldsymbol{u}}{(2 \pi)^{3} 2 u^{0}} \int \frac{d^{3} \boldsymbol{v}}{(2 \pi)^{3} 2 v^{0}} \times \\
& T\left[\left(S_{\mu \nu} T^{\mu \nu} e^{-i(s+t) x} a_{\boldsymbol{s}} a_{\boldsymbol{t}}+S_{\mu \nu} \hat{T}^{\mu \nu} e^{-i(s-t) x} a_{\boldsymbol{s}} a_{\boldsymbol{t}}^{\dagger}+\right.\right. \\
& \left.\hat{S}_{\rho \sigma} T^{\rho \sigma} e^{-i(t-s) x} a_{\boldsymbol{s}}^{\dagger} a_{\boldsymbol{t}}+\hat{S}_{\rho \sigma} \hat{T}^{\rho \sigma} e^{i(s+t) x} a_{\boldsymbol{s}}^{\dagger} a_{\boldsymbol{t}}^{\dagger}\right) \times \\
& \left(U_{\mu \nu} V^{\mu \nu} e^{-i(u+v) y} a_{\boldsymbol{u}} a_{\boldsymbol{v}}+U_{\mu \nu} \hat{V}^{\mu \nu} e^{-i(u-v) y} a_{\boldsymbol{u}} a_{\boldsymbol{v}}^{\dagger}+\right. \\
& \left.\left.\hat{U}_{\rho \sigma} V^{\rho \sigma} e^{-i(v-u) y} a_{\boldsymbol{u}}^{\dagger} a_{\boldsymbol{v}}+\hat{U}_{\rho \sigma} \hat{V}^{\rho \sigma} e^{i(u+v) y} a_{\boldsymbol{u}}^{\dagger} a_{\boldsymbol{v}}^{\dagger}\right)\right] .
\end{aligned}
$$

Since we focus on only two-body-two-body interactions, the relevant S-matrix (including two creation and two annihilation operators) is expressed as

$$
\begin{aligned}
S_{2 \rightarrow 2}^{(2)}= & \left(-\frac{1}{4} \frac{g}{M}\right)^{2} \frac{i}{2}(2 \pi)^{4} \int \frac{d^{3} \boldsymbol{s}}{(2 \pi)^{3} 2 s^{0}} \int \frac{d^{3} \boldsymbol{t}}{(2 \pi)^{3} 2 t^{0}} \int \frac{d^{3} \boldsymbol{u}}{(2 \pi)^{3} 2 u^{0}} \int \frac{d^{3} \boldsymbol{v}}{(2 \pi)^{3} 2 v^{0}} \times \\
& \left(G_{-s,-t} \delta^{4}(-u-v+s+t) S_{\mu \nu} T^{\mu \nu} \hat{U}_{\rho \sigma} \hat{V}^{\rho \sigma} a_{\boldsymbol{u}}^{\dagger} a_{\boldsymbol{v}}^{\dagger} a_{\boldsymbol{s}} a_{\boldsymbol{t}}+\right. \\
& G_{-s,+t} \delta^{4}(+u-v+s-t) S_{\mu \nu} \hat{T}^{\mu \nu} U_{\rho \sigma} \hat{V}^{\rho \sigma} a_{\boldsymbol{t}}^{\dagger} a_{\boldsymbol{v}}^{\dagger} a_{\boldsymbol{s}} a_{\boldsymbol{u}}+ \\
& G_{-s,+t} \delta^{4}(-u+v+s-t) S_{\mu \nu} \hat{T}^{\mu \nu} \hat{U}_{\rho \sigma} V^{\rho \sigma} a_{\boldsymbol{t}}^{\dagger} a_{\boldsymbol{u}}^{\dagger} a_{\boldsymbol{s}} a_{\boldsymbol{v}}+ \\
& G_{+s,-t} \delta^{4}(+u-v-s+t) \hat{S}_{\mu \nu} T^{\mu \nu} U_{\rho \sigma} \hat{V}^{\rho \sigma} a_{\boldsymbol{s}}^{\dagger} a_{\boldsymbol{v}}^{\dagger} a_{\boldsymbol{t}} a_{\boldsymbol{u}}+ \\
& G_{+s,-t} \delta^{4}(-u+v-s+t) \hat{S}_{\mu \nu} T^{\mu \nu} \hat{U}_{\rho \sigma} V^{\rho \sigma} a_{\boldsymbol{s}}^{\dagger} a_{\boldsymbol{u}}^{\dagger} a_{\boldsymbol{t}} a_{\boldsymbol{v}}+ \\
& \left.G_{+s,+t} \delta^{4}(+u+v-s-t) \hat{S}_{\mu \nu} \hat{T}^{\mu \nu} U_{\rho \sigma} V^{\rho \sigma} a_{\boldsymbol{s}}^{\dagger} a_{\boldsymbol{t}}^{\dagger} a_{\boldsymbol{u}} a_{\boldsymbol{v}}\right),
\end{aligned}
$$

where $G_{i, j} \equiv\left(m^{2}-(i+j)^{2}\right)^{-1}$ indicate corresponding propagators.

Let us recall the definition of the coherent state [20]:

$$
\left.\left|N_{\boldsymbol{p}}\right\rangle\right\rangle \equiv \exp \left(-N_{\boldsymbol{p}} / 2\right) \sum_{n=0}^{\infty} \frac{N_{\boldsymbol{p}}^{n / 2}}{\sqrt{n !}}\left|n_{\boldsymbol{p}}\right\rangle,
$$

where $\left|n_{\boldsymbol{p}}\right\rangle$ is the normalized state of $n$ photons

$$
\left|n_{\boldsymbol{p}}\right\rangle=\frac{1}{\sqrt{n !}}\left(a_{\boldsymbol{p}}^{\dagger}\right)^{n}|0\rangle
$$


with the creation operator $a_{\boldsymbol{p}}^{\dagger}$ of photons that share a common momentum $\boldsymbol{p}$ and a common polarization state over different number states. The following relations on the coherent state

$$
\left\langle\left\langle N_{\boldsymbol{p}} \mid N_{\boldsymbol{p}}\right\rangle\right\rangle=1
$$

and

$$
\left\langle\left\langle N_{\boldsymbol{p}}|n| N_{\boldsymbol{p}}\right\rangle\right\rangle=\left\langle\left\langle N_{\boldsymbol{p}}\left|\left(a_{\boldsymbol{p}}^{\dagger} a_{\boldsymbol{p}}\right)\right| N_{\boldsymbol{p}}\right\rangle\right\rangle=N_{\boldsymbol{p}},
$$

give us basic properties with respect to the creation and annihilation operators:

$$
\left.\left.a_{\boldsymbol{p}}\left|N_{\boldsymbol{p}}\right\rangle\right\rangle=\sqrt{N_{\boldsymbol{p}}}\left|N_{\boldsymbol{p}}\right\rangle\right\rangle, \quad \text { and } \quad\left\langle\left\langle N_{\boldsymbol{p}}\right| a_{\boldsymbol{p}}^{\dagger}=\sqrt{N_{\boldsymbol{p}}}\left\langle\left\langle N_{\boldsymbol{p}}\right|\right.\right.
$$

We first consider a search for signal photons $p_{3}$ via the scattering process $p_{1}+p_{2} \rightarrow p_{3}+p_{4}$ by supplying coherent fields $\left.\left.\left|N_{\boldsymbol{p}_{1}}\right\rangle\right\rangle,\left|N_{\boldsymbol{p}_{2}}\right\rangle\right\rangle$ and $\left.\left|N_{\boldsymbol{p}_{4}}\right\rangle\right\rangle$. We then introduce initial and final states, respectively, as follows:

$$
\begin{aligned}
|\Omega\rangle & \left.\left.\left.\equiv\left|N_{\boldsymbol{p}_{1}}\right\rangle\right\rangle\left|N_{\boldsymbol{p}_{2}}\right\rangle\right\rangle\left|N_{\boldsymbol{p}_{4}}\right\rangle\right\rangle|0\rangle, \text { and } \\
\left\langle\Omega^{\prime}\right| & \equiv\left\langle\langle N _ { \boldsymbol { p } _ { 1 } } | \left\langle\langle N _ { \boldsymbol { p } _ { 2 } } | \left\langle\left\langle N_{\boldsymbol{p}_{4}}\right|\left\langle 1_{\boldsymbol{p}_{3}}\right|=\langle\Omega| a_{\boldsymbol{p}_{3}} .\right.\right.\right.
\end{aligned}
$$

The two-body transition amplitude $\left\langle\Omega^{\prime}\left|S_{2 \rightarrow 2}^{(2)}\right| \Omega\right\rangle$ contains the common operator products $a_{i}^{\dagger} a_{j}^{\dagger} a_{\boldsymbol{k}} a_{\boldsymbol{l}}$. We then separately evaluate the contractions with coherent bra- and ket-states, respectively, as follows:

$$
\begin{aligned}
\left\langle\Omega^{\prime}\right| a_{\boldsymbol{i}}^{\dagger} a_{\boldsymbol{j}}^{\dagger}= & \sqrt{N_{p_{1}}} \hat{\delta}^{3}\left(\boldsymbol{p}_{1}-\boldsymbol{i}\right)\left\{\left\langle\Omega^{\prime}\right| \sqrt{N_{p_{1}}} \hat{\delta}^{3}\left(\boldsymbol{p}_{1}-\boldsymbol{j}\right)+\left\langle\Omega^{\prime}\right| \sqrt{N_{p_{2}}} \hat{\delta}^{3}\left(\boldsymbol{p}_{2}-\boldsymbol{j}\right)+\right. \\
& \left.\left\langle\Omega^{\prime}\right| \sqrt{N_{p_{4}}} \hat{\delta}^{3}\left(\boldsymbol{p}_{4}-\boldsymbol{j}\right)+\langle\Omega| 1 \hat{\delta}^{3}\left(\boldsymbol{p}_{3}-\boldsymbol{j}\right)\right\}+\sqrt{N_{p_{2}}} \hat{\delta}^{3}\left(\boldsymbol{p}_{2}-\boldsymbol{i}\right) \times \\
& \left\{\left\langle\Omega^{\prime}\right| \sqrt{N_{p_{1}}} \hat{\delta}^{3}\left(\boldsymbol{p}_{1}-\boldsymbol{j}\right)+\left\langle\Omega^{\prime}\right| \sqrt{N_{p_{2}}} \hat{\delta}^{3}\left(\boldsymbol{p}_{2}-\boldsymbol{j}\right)+\right. \\
& \left.\left\langle\Omega^{\prime}\right| \sqrt{N_{p_{4}}} \hat{\delta}^{3}\left(\boldsymbol{p}_{4}-\boldsymbol{j}\right)+\langle\Omega| 1 \hat{\delta}^{3}\left(\boldsymbol{p}_{3}-\boldsymbol{j}\right)\right\}+\sqrt{N_{p_{4}}} \hat{\delta}^{3}\left(\boldsymbol{p}_{4}-\boldsymbol{i}\right) \times \\
& \left\{\left\langle\Omega^{\prime}\right| \sqrt{N_{p_{1}}} \hat{\delta}^{3}\left(\boldsymbol{p}_{1}-\boldsymbol{j}\right)+\left\langle\Omega^{\prime}\right| \sqrt{N_{p_{2}}} \hat{\delta}^{3}\left(\boldsymbol{p}_{2}-\boldsymbol{j}\right)+\right. \\
& \left.\left\langle\Omega^{\prime}\right| \sqrt{N_{p_{4}}} \hat{\delta}^{3}\left(\boldsymbol{p}_{4}-\boldsymbol{j}\right)+\langle\Omega| 1 \hat{\delta}^{3}\left(\boldsymbol{p}_{3}-\boldsymbol{j}\right)\right\}+1 \hat{\delta}^{3}\left(\boldsymbol{p}_{3}-\boldsymbol{i}\right) \times \\
& \left\{\langle\Omega| \sqrt{N_{p_{1}}} \hat{\delta}^{3}\left(\boldsymbol{p}_{1}-\boldsymbol{j}\right)+\langle\Omega| \sqrt{N_{p_{2}}} \hat{\delta}^{3}\left(\boldsymbol{p}_{2}-\boldsymbol{j}\right)+\right. \\
& \left.\langle\Omega| \sqrt{N_{p_{4}}} \hat{\delta}^{3}\left(\boldsymbol{p}_{4}-\boldsymbol{j}\right)+\langle\Omega| a_{\boldsymbol{j}}^{\dagger}\right\},
\end{aligned}
$$

where the last term vanishes because $\langle 0| a_{\boldsymbol{p}}^{\dagger}=0$, and

$$
\begin{aligned}
a_{\boldsymbol{k}} a_{\boldsymbol{l}}|\Omega\rangle= & \sqrt{N_{p_{1}}} \hat{\delta}^{3}\left(\boldsymbol{p}_{1}-\boldsymbol{l}\right)\left\{\sqrt{N_{p_{1}}} \hat{\delta}^{3}\left(\boldsymbol{p}_{1}-\boldsymbol{k}\right)|\Omega\rangle+\right. \\
& \left.\sqrt{N_{p_{2}}} \hat{\delta}^{3}\left(\boldsymbol{p}_{2}-\boldsymbol{k}\right)|\Omega\rangle+\sqrt{N_{p_{4}}} \hat{\delta}^{3}\left(\boldsymbol{p}_{4}-\boldsymbol{k}\right)|\Omega\rangle\right\}+ \\
& \sqrt{N_{p_{2}}} \hat{\delta}^{3}\left(\boldsymbol{p}_{2}-\boldsymbol{l}\right)\left\{\sqrt{N_{p_{1}}} \hat{\delta}^{3}\left(\boldsymbol{p}_{1}-\boldsymbol{k}\right)|\Omega\rangle+\right. \\
& \left.\sqrt{N_{p_{2}}} \hat{\delta}^{3}\left(\boldsymbol{p}_{2}-\boldsymbol{k}\right)|\Omega\rangle+\sqrt{N_{p_{4}}} \hat{\delta}^{3}\left(\boldsymbol{p}_{4}-\boldsymbol{k}\right)|\Omega\rangle\right\}+ \\
& \sqrt{N_{p_{4}}} \hat{\delta}^{3}\left(\boldsymbol{p}_{4}-\boldsymbol{l}\right)\left\{\sqrt{N_{p_{1}}} \hat{\delta}^{3}\left(\boldsymbol{p}_{1}-\boldsymbol{k}\right)|\Omega\rangle+\right. \\
& \left.\sqrt{N_{p_{2}}} \hat{\delta}^{3}\left(\boldsymbol{p}_{2}-\boldsymbol{k}\right)|\Omega\rangle+\sqrt{N_{p_{4}}} \hat{\delta}^{3}\left(\boldsymbol{p}_{4}-\boldsymbol{k}\right)|\Omega\rangle\right\} .
\end{aligned}
$$

Because the search window is designed for the scattering process $p_{1}+p_{2} \rightarrow p_{3}+p_{4}$, the consistent transition amplitude that satisfies the combination of the initial and final state 
momenta is limited to

$$
\begin{aligned}
\left\langle\Omega^{\prime}\left|a_{\boldsymbol{i}}^{\dagger} a_{\boldsymbol{j}}^{\dagger} a_{\boldsymbol{k}} a_{\boldsymbol{l}}\right| \Omega\right\rangle= & \left\{\sqrt{N_{p_{4}}} \hat{\delta}^{3}\left(\boldsymbol{p}_{4}-\boldsymbol{i}\right) 1 \hat{\delta}^{3}\left(\boldsymbol{p}_{3}-\boldsymbol{j}\right)+1 \hat{\delta}^{3}\left(\boldsymbol{p}_{3}-\boldsymbol{i}\right) \sqrt{N_{p_{4}}} \hat{\delta}^{3}\left(\boldsymbol{p}_{4}-\boldsymbol{j}\right)\right\} \times \\
& \left\{\sqrt{N_{p_{1}}} \hat{\delta}^{3}\left(\boldsymbol{p}_{1}-\boldsymbol{l}\right) \sqrt{N_{p_{2}}} \hat{\delta}^{3}\left(\boldsymbol{p}_{2}-\boldsymbol{k}\right)+\sqrt{N_{p_{2}}} \hat{\delta}^{3}\left(\boldsymbol{p}_{2}-\boldsymbol{l}\right) \sqrt{N_{p_{1}}} \hat{\delta}^{3}\left(\boldsymbol{p}_{1}-\boldsymbol{k}\right)\right\}\langle\Omega \mid \Omega\rangle \\
= & \sqrt{N_{p_{1}}} \sqrt{N_{p_{2}}} \sqrt{N_{p_{4}}}\left\{\hat{\delta}^{3}\left(\boldsymbol{p}_{4}-\boldsymbol{i}\right) \hat{\delta}^{3}\left(\boldsymbol{p}_{3}-\boldsymbol{j}\right) \hat{\delta}^{3}\left(\boldsymbol{p}_{2}-\boldsymbol{k}\right) \hat{\delta}^{3}\left(\boldsymbol{p}_{1}-\boldsymbol{l}\right)+\right. \\
& \hat{\delta}^{3}\left(\boldsymbol{p}_{4}-\boldsymbol{i}\right) \hat{\delta}^{3}\left(\boldsymbol{p}_{3}-\boldsymbol{j}\right) \hat{\delta}^{3}\left(\boldsymbol{p}_{1}-\boldsymbol{k}\right) \hat{\delta}^{3}\left(\boldsymbol{p}_{2}-\boldsymbol{l}\right)+ \\
& \hat{\delta}^{3}\left(\boldsymbol{p}_{3}-\boldsymbol{i}\right) \hat{\delta}^{3}\left(\boldsymbol{p}_{4}-\boldsymbol{j}\right) \hat{\delta}^{3}\left(\boldsymbol{p}_{2}-\boldsymbol{k}\right) \hat{\delta}^{3}\left(\boldsymbol{p}_{1}-\boldsymbol{l}\right)+ \\
& \left.\hat{\delta}^{3}\left(\boldsymbol{p}_{3}-\boldsymbol{i}\right) \hat{\delta}^{3}\left(\boldsymbol{p}_{4}-\boldsymbol{j}\right) \hat{\delta}^{3}\left(\boldsymbol{p}_{1}-\boldsymbol{k}\right) \hat{\delta}^{3}\left(\boldsymbol{p}_{2}-\boldsymbol{l}\right)\right\},
\end{aligned}
$$

where $\langle\Omega \mid \Omega\rangle=1$ is used.

By assigning any of $\boldsymbol{i}, \boldsymbol{j}, \boldsymbol{k}$, and $\boldsymbol{l}$ in eq. (A.18) to any of $\boldsymbol{s}, \boldsymbol{t}, \boldsymbol{u}, \boldsymbol{v}$ in eq. (A.9), the two-body transition amplitude can be expressed as

$$
\begin{aligned}
& \left\langle\Omega^{\prime}\left|S_{2 \rightarrow 2}^{(2)}\right| \Omega\right\rangle=\left(-\frac{1}{4} \frac{g}{M}\right)^{2} \frac{i}{2}(2 \pi)^{4} \delta^{(4)}\left(p_{1}+p_{2}-p_{3}-p_{4}\right) \sqrt{N_{p_{1}}} \sqrt{N_{p_{2}}} \sqrt{N_{p_{4}}} \times \\
& \left(\frac{8\left(P_{1} P_{2}\right)\left(\hat{P}_{3} \hat{P}_{4}\right)}{m^{2}-\left(p_{1}+p_{2}\right)^{2}}+\frac{4\left(P_{2} \hat{P}_{4}\right)\left(P_{1} \hat{P}_{3}\right)}{m^{2}-\left(p_{2}-p_{4}\right)^{2}}+\frac{4\left(P_{2} \hat{P}_{3}\right)\left(P_{1} \hat{P}_{4}\right)}{m^{2}-\left(p_{2}-p_{3}\right)^{2}}+\frac{4\left(P_{1} \hat{P}_{4}\right)\left(P_{2} \hat{P}_{3}\right)}{m^{2}-\left(p_{1}-p_{4}\right)^{2}}+\frac{4\left(P_{1} \hat{P}_{3}\right)\left(P_{2} \hat{P}_{4}\right)}{m^{2}-\left(p_{1}-p_{3}\right)^{2}}\right),
\end{aligned}
$$

where subscripts have been omitted in the momentum-polarization tensors such as $(S \hat{T}) \equiv S_{\mu \nu} \hat{T}^{\mu \nu}$.

From the experimental point of view, it is also useful to consider the case $p_{1}+p_{1} \rightarrow$ $p_{3}+p_{4}$, where the initial state photons are from a degenerate state, because the number of incident beams can be reduced from two to one in an experimental setup. For the degenerate case, we define the initial and final states, respectively, as follows:

$$
\begin{aligned}
|\Omega\rangle & \left.\left.\equiv\left|N_{\boldsymbol{p}_{1}}\right\rangle\right\rangle\left|N_{\boldsymbol{p}_{4}}\right\rangle\right\rangle|0\rangle, \text { and } \\
\left\langle\Omega^{\prime}\right| & \equiv\left\langle\langle N _ { \boldsymbol { p } _ { 1 } } | \left\langle\left\langle N_{\boldsymbol{p}_{4}}\right|\left\langle 1_{\boldsymbol{p}_{3}}\right|=\langle\Omega| a_{\boldsymbol{p}_{3}} .\right.\right.
\end{aligned}
$$

For the evaluation of the two-body transition amplitude $\left\langle\Omega^{\prime}\left|S_{2 \rightarrow 2}^{(2)}\right| \Omega\right\rangle$ containing $a_{\boldsymbol{i}}^{\dagger} a_{\boldsymbol{j}}^{\dagger} a_{\boldsymbol{k}} a_{\boldsymbol{l}}$, we again separately evaluate the contractions with coherent bra- and ket-states, respectively, as follows:

$$
\begin{aligned}
\left\langle\Omega^{\prime}\right| a_{\boldsymbol{i}}^{\dagger} a_{\boldsymbol{j}}^{\dagger}= & \sqrt{N_{p_{1}}} \hat{\delta}^{3}\left(\boldsymbol{p}_{1}-\boldsymbol{i}\right)\left\{\left\langle\Omega^{\prime}\right| \sqrt{N_{p_{1}}} \hat{\delta}^{3}\left(\boldsymbol{p}_{1}-\boldsymbol{j}\right)+\left\langle\Omega^{\prime}\right| \sqrt{N_{p_{4}}} \hat{\delta}^{3}\left(\boldsymbol{p}_{4}-\boldsymbol{j}\right)+\langle\Omega| 1 \hat{\delta}^{3}\left(\boldsymbol{p}_{3}-\boldsymbol{j}\right)\right\}+ \\
& \sqrt{N_{p_{4}}} \hat{\delta}^{3}\left(\boldsymbol{p}_{4}-\boldsymbol{i}\right)\left\{\left\langle\Omega^{\prime}\right| \sqrt{N_{p_{1}}} \hat{\delta}^{3}\left(\boldsymbol{p}_{1}-\boldsymbol{j}\right)+\left\langle\Omega^{\prime}\right| \sqrt{N_{p_{4}}} \hat{\delta}^{3}\left(\boldsymbol{p}_{4}-\boldsymbol{j}\right)+\langle\Omega| 1 \hat{\delta}^{3}\left(\boldsymbol{p}_{3}-\boldsymbol{j}\right)\right\}+ \\
& 1 \hat{\delta}^{3}\left(\boldsymbol{p}_{3}-\boldsymbol{i}\right)\left\{\langle\Omega| \sqrt{N_{p_{1}}} \hat{\delta}^{3}\left(\boldsymbol{p}_{1}-\boldsymbol{j}\right)+\langle\Omega| \sqrt{N_{p_{4}}} \hat{\delta}^{3}\left(\boldsymbol{p}_{4}-\boldsymbol{j}\right)+\langle\Omega| a_{\boldsymbol{j}}^{\dagger}\right\},
\end{aligned}
$$

where the last term vanishes because $\langle 0| a_{p}^{\dagger}=0$, and

$$
\begin{aligned}
a_{\boldsymbol{k}} a_{\boldsymbol{l}}|\Omega\rangle= & \sqrt{N_{p_{1}}} \hat{\delta}^{3}\left(\boldsymbol{p}_{1}-\boldsymbol{l}\right)\left\{\sqrt{N_{p_{1}}} \hat{\delta}^{3}\left(\boldsymbol{p}_{1}-\boldsymbol{k}\right)|\Omega\rangle+\sqrt{N_{p_{4}}} \hat{\delta}^{3}\left(\boldsymbol{p}_{4}-\boldsymbol{k}\right)|\Omega\rangle\right\}+ \\
& \sqrt{N_{p_{4}}} \hat{\delta}^{3}\left(\boldsymbol{p}_{4}-\boldsymbol{l}\right)\left\{\sqrt{N_{p_{1}}} \hat{\delta}^{3}\left(\boldsymbol{p}_{1}-\boldsymbol{k}\right)|\Omega\rangle+\sqrt{N_{p_{4}}} \hat{\delta}^{3}\left(\boldsymbol{p}_{4}-\boldsymbol{k}\right)|\Omega\rangle\right\} .
\end{aligned}
$$




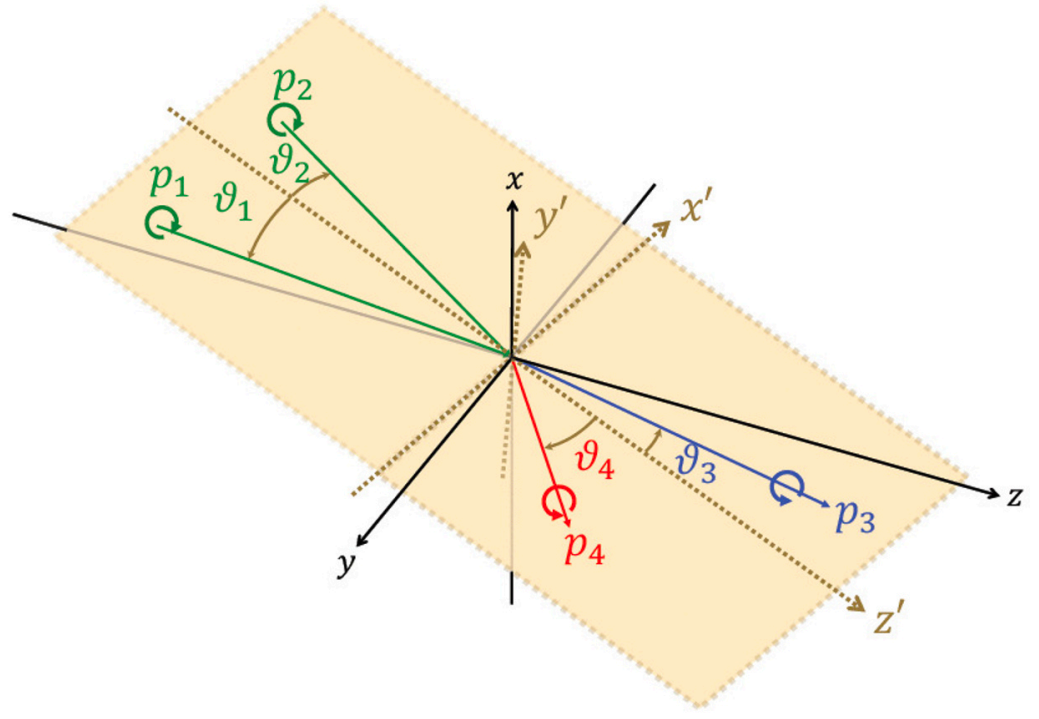

Figure 4. Kinematical parameters in asymmetric-incident and non-coaxial geometry. Primed coordinates $\left(x^{\prime}, y^{\prime}, z^{\prime}\right)$, where the total transverse momentum of an arbitrary selected pair of incident photons within the creation beam vanishes, are configurable with respect to the fixed laboratory coordinate $(x, y, z)$ to which the focused beam fields are physically mapped. The kinematical parameters used to derive the scattering amplitude are all based on these primed coordinates.

The consistent transition amplitude that satisfies the combination of the initial and final state momenta in the degenerate case is expressed as

$$
\begin{aligned}
\left\langle\Omega^{\prime}\left|a_{i}^{\dagger} a_{\boldsymbol{j}}^{\dagger} a_{\boldsymbol{k}} a_{\boldsymbol{l}}\right| \Omega\right\rangle= & \left\{\sqrt{N_{p_{4}}} \hat{\delta}^{3}\left(\boldsymbol{p}_{4}-\boldsymbol{i}\right) 1 \hat{\delta}^{3}\left(\boldsymbol{p}_{3}-\boldsymbol{j}\right)+1 \hat{\delta}^{3}\left(\boldsymbol{p}_{3}-\boldsymbol{i}\right) \sqrt{N_{p_{4}}} \hat{\delta}^{3}\left(\boldsymbol{p}_{4}-\boldsymbol{j}\right)\right\} \times \\
& \sqrt{N_{p_{1}}} \hat{\delta}^{3}\left(\boldsymbol{p}_{1}-\boldsymbol{l}\right) \sqrt{N_{p_{1}}} \hat{\delta}^{3}\left(\boldsymbol{p}_{1}-\boldsymbol{k}\right)\langle\Omega \mid \Omega\rangle \\
= & \sqrt{N_{p_{1}}} \sqrt{N_{p_{1}}} \sqrt{N_{p_{4}}}\left\{\hat{\delta}^{3}\left(\boldsymbol{p}_{4}-\boldsymbol{i}\right) \hat{\delta}^{3}\left(\boldsymbol{p}_{3}-\boldsymbol{j}\right) \hat{\delta}^{3}\left(\boldsymbol{p}_{1}-\boldsymbol{k}\right) \hat{\delta}^{3}\left(\boldsymbol{p}_{1}-\boldsymbol{l}\right)+\right. \\
& \left.\hat{\delta}^{3}\left(\boldsymbol{p}_{3}-\boldsymbol{i}\right) \hat{\delta}^{3}\left(\boldsymbol{p}_{4}-\boldsymbol{j}\right) \hat{\delta}^{3}\left(\boldsymbol{p}_{1}-\boldsymbol{k}\right) \hat{\delta}^{3}\left(\boldsymbol{p}_{1}-\boldsymbol{l}\right)\right\}
\end{aligned}
$$

where $\langle\Omega \mid \Omega\rangle=1$ is substituted.

Again assigning any of $\boldsymbol{i}, \boldsymbol{j}, \boldsymbol{k}$, and $\boldsymbol{l}$ in eq. (A.23) to any of $\boldsymbol{s}, \boldsymbol{t}, \boldsymbol{u}$, and $\boldsymbol{v}$ in eq. (A.9), the two-body transition amplitude is expressed as

$$
\begin{aligned}
\left\langle\Omega^{\prime}\left|S_{2 \rightarrow 2}^{(2)}\right| \Omega\right\rangle= & \left(-\frac{1}{4} \frac{g}{M}\right)^{2} \frac{i}{2}(2 \pi)^{4} \delta^{(4)}\left(p_{1}+p_{1}-p_{3}-p_{4}\right) \sqrt{N_{p_{1}}} \sqrt{N_{p_{1}}} \sqrt{N_{p_{4}}} \times \\
& \left(\frac{4\left(P_{1} P_{1}\right)\left(\hat{P}_{3} \hat{P}_{4}\right)}{m^{2}-\left(p_{1}+p_{1}\right)^{2}}+\frac{4\left(P_{1} \hat{P}_{4}\right)\left(P_{1} \hat{P}_{3}\right)}{m^{2}-\left(p_{1}-p_{4}\right)^{2}}+\frac{4\left(P_{1} \hat{P}_{3}\right)\left(P_{1} \hat{P}_{4}\right)}{m^{2}-\left(p_{1}-p_{3}\right)^{2}}\right)
\end{aligned}
$$

We take special note that the degenerate case may also be interpreted as a special case of the non-degenerate case by reducing the average number of $p_{1}$ photons from $N_{p_{1}}$ to $N_{p_{1}} / 2$ due to the equal split into two identical beams and equating 1 and 2 in the subscripts in eq. (A.19). 


\section{A.2 Kinematics in asymmetric-incident and non-coaxial geometry in QPS}

As illustrated in figure 4, we extend the scattering formulation to the most general scattering geometry, which is asymmetric-incident and non-coaxial scattering in QPS. For a selected pair of incident waves, $p_{1}$ and $p_{2}$, from a coherent creation beam, we can always define an axis $z^{\prime}$ around which the total transverse momentum, $p_{T}$, of the two incident waves becomes zero and an axis $x^{\prime}$ normal to the $z^{\prime}$-axis on the reaction plane that includes the two wave vectors, as shown in figure 4 . On this $x^{\prime}-z^{\prime}$ plane, referred to as the $z e r o-p_{T}$ coordinate, the scattering amplitude is greatly simplified because the emission angles of final state waves $p_{3}$ and $p_{4}$ become axially symmetric around the $z^{\prime}$-axis. The labels for energies are common to both the zero- $p_{T}$ coordinate and the laboratory coordinate defined in terms of the $x, y$, and $z$-axes, while angular or momentum labels are valid only for the zero- $p_{T}$ coordinate. In the following subsections, for simplicity, we use kinematical parameters defined in the zero- $p_{T}$ coordinate, even when the prime symbol is not written, except where laboratory coordinates are explicitly specified.

With the energies of four photons $\omega_{i}$ and scattering angles $\vartheta_{i}$ for initial $i=1,2$ and final $i=3,4$ states in the zero- $p_{T}$ coordinate, four-momenta are defined as follows:

$$
\begin{aligned}
& p_{1}=\left(\omega_{1}, \quad \omega_{1} \sin \vartheta_{1}, 0, \omega_{1} \cos \vartheta_{1}\right), \\
& p_{2}=\left(\omega_{2},-\omega_{2} \sin \vartheta_{2}, 0, \omega_{2} \cos \vartheta_{2}\right), \\
& p_{3}=\left(\omega_{3}, \quad \omega_{3} \sin \vartheta_{3}, 0, \omega_{3} \cos \vartheta_{3}\right), \\
& p_{4}=\left(\omega_{4},-\omega_{4} \sin \vartheta_{4}, 0, \omega_{4} \cos \vartheta_{4}\right) .
\end{aligned}
$$

For later convenience, a bisecting angle $\vartheta_{b}$ is introduced, with the meaning

$$
\vartheta_{b} \equiv \frac{\vartheta_{1}+\vartheta_{2}}{2} .
$$

The energy-momentum conservation equalities are

$$
\begin{aligned}
\omega_{1}+\omega_{2} & =\omega_{3}+\omega_{4} \\
\omega_{1} \cos \vartheta_{1}+\omega_{2} \cos \vartheta_{2} & =\omega_{3} \cos \vartheta_{3}+\omega_{4} \cos \vartheta_{4} \equiv \omega_{z} \\
\omega_{1} \sin \vartheta_{1}-\omega_{2} \sin \vartheta_{2} & =\omega_{3} \sin \vartheta_{3}-\omega_{4} \sin \vartheta_{4} \equiv \omega_{x} .
\end{aligned}
$$

The corresponding center-of-mass energy, $E_{\mathrm{cms}}$, is then expressed as

$$
E_{\mathrm{cms}}=\sqrt{2 \omega_{1} \omega_{2}\left\{1-\cos \left(\vartheta_{1}+\vartheta_{2}\right)\right\}}=2 \sqrt{\omega_{1} \omega_{2}} \sin \vartheta_{b} .
$$

We then define the linear polarization vectors as

$$
\begin{aligned}
& \boldsymbol{e}_{i}^{(1)}=(0,1,0), \\
& \boldsymbol{e}_{1}^{(2)}=\left(-\cos \vartheta_{1}, 0, \sin \vartheta_{1}\right), \\
& \boldsymbol{e}_{2}^{(2)}=\left(-\cos \vartheta_{2}, 0,-\sin \vartheta_{2}\right), \\
& \boldsymbol{e}_{3}^{(2)}=\left(-\cos \vartheta_{3}, 0, \sin \vartheta_{3}\right), \\
& \boldsymbol{e}_{4}^{(2)}=\left(-\cos \vartheta_{4}, 0,-\sin \vartheta_{4}\right) .
\end{aligned}
$$


With the linear polarization vectors, we also can define circular polarization states:

$$
\begin{aligned}
\boldsymbol{e}_{i}^{R} & =\frac{1}{\sqrt{2}}\left(\boldsymbol{e}_{i}^{(1)}+i \boldsymbol{e}_{i}^{(2)}\right), \\
\boldsymbol{e}_{i}^{L} & =\frac{1}{\sqrt{2}}\left(\boldsymbol{e}_{i}^{(1)}-i \boldsymbol{e}_{i}^{(2)}\right) .
\end{aligned}
$$

Given these definitions, we can evaluate the momentum-polarization tensors included in eq. (A.19) for the circular polarization case as follows

$$
\begin{aligned}
& \left(P_{i} P_{j}\right)=\left(\hat{P}_{i} \hat{P}_{j}\right)=2 \omega_{i} \omega_{j}\left(1-\cos \left(\vartheta_{i}+\vartheta_{j}\right)\right) \\
& \left(P_{i} \hat{P}_{j}\right)=\left(\hat{P}_{i} P_{j}\right)=0 .
\end{aligned}
$$

\section{A.3 Lorentz-invariant scattering amplitude including a resonance state}

Here we are particularly interested in formulating a Lorentz-invariant scattering amplitude for circular polarization states, because the states describe naturally interpretable angular momenta of photons with respect to any directions of the photon momenta. We denote a sequence of four-photon circular polarization states as a subscript $S \equiv a b c d$ with $a, b, c, d=$ $R$ (right-handed) or $L$ (left-handed). From the following definition for the transition amplitude

$$
\left\langle\Omega^{\prime}\left|S_{2 \rightarrow 2}^{(2)}\right| \Omega\right\rangle=\sqrt{N_{p_{1}}} \sqrt{N_{p_{2}}} \sqrt{N_{p_{4}}} i(2 \pi)^{4} \delta^{(4)}\left(p_{1}+p_{2}-p_{3}-p_{4}\right) \mathcal{M}_{S},
$$

the Lorentz-invariant scattering amplitude $\mathcal{M}_{S}$ can be expressed as

$$
\mathcal{M}_{S}=\frac{1}{4}\left(\frac{g}{M}\right)^{2} \frac{\left(P_{1} P_{2}\right)\left(\hat{P}_{3} \hat{P}_{4}\right)}{m^{2}-\left(p_{1}+p_{2}\right)^{2}}
$$

from eq. (A.19) by taking eq. (A.31) into account, where we exclude the beam-relevant factor in the definition of $\mathcal{M}_{S}$ so as to decouple the dynamics from the experimental factor caused by the coherent beam intensity. With eq. (A.31), the vertex factors in the numerator of $\mathcal{M}_{S}$ are expressed as

$$
\begin{aligned}
& \left(P_{1} P_{2}\right)=2 \omega_{1} \omega_{2}\left(1-\cos \left(\vartheta_{1}+\vartheta_{2}\right)\right) \\
& \left(\hat{P}_{3} \hat{P}_{4}\right)=2 \omega_{3} \omega_{4}\left(1-\cos \left(\vartheta_{3}+\vartheta_{4}\right)\right) .
\end{aligned}
$$

Since energy-momentum conservation requires $\left(p_{1}+p_{2}\right)^{2}=\left(p_{3}+p_{4}\right)^{2}$, we can describe the amplitude applicable to both $S=L L R R$ and $R R L L$ in terms of only the initial state variables, as follows:

$$
\begin{aligned}
\mathcal{M}_{S} & =\left(\frac{g}{M}\right)^{2} \frac{\left(\omega_{1} \omega_{2}\left(\cos \left(\vartheta_{1}+\vartheta_{2}\right)-1\right)\right)^{2}}{m^{2}-2 \omega_{1} \omega_{2}\left(1-\cos \left(\vartheta_{1}+\vartheta_{2}\right)\right)} \\
& =\left(\frac{g}{M}\right)^{2} \frac{\left(2 \omega_{1} \omega_{2} \sin ^{2} \vartheta_{b}\right)^{2}}{m^{2}-4 \omega_{1} \omega_{2} \sin ^{2} \vartheta_{b}}
\end{aligned}
$$

where eq. (A.26) is substituted for the second relation. To implement energy fluctuations in the initial state of two photons chosen from a solo coherent beam around its central energy $\omega_{c}$, we introduce two independent parameters $s_{1}$ and $s_{2}$, as follows:

$$
\omega_{1}=s_{1} \omega_{\mathrm{c}}, \quad \omega_{2}=s_{2} \omega_{\mathrm{c}}
$$


We then define a resonance energy $\omega_{r}$ satisfying $E_{\mathrm{cms}}=m$ as

$$
\omega_{r}^{2} \equiv \frac{m^{2}}{4 s_{1} s_{2} \sin ^{2} \vartheta_{b}} .
$$

Because the exchanged scalar field is (in principle) an unstable particle, we introduce a decay rate $\Gamma$, which is defined as $[5]$

$$
\Gamma=\frac{1}{16 \pi}\left(\frac{g}{M}\right)^{2} m^{3} .
$$

This causes a change in the mass square as $m^{2} \rightarrow(m-i \Gamma / 2)^{2} \approx m^{2}-i m \Gamma$. Therefore, the denominator $\mathcal{D}$ in eq. (A.35) is expressed as

$$
\begin{aligned}
\mathcal{D} & \approx-4 s_{1} s_{2} \omega_{\mathrm{c}}^{2} \sin ^{2} \vartheta_{b}+m^{2}-i \Gamma m \\
& =-4 s_{1} s_{2} \omega_{\mathrm{c}}^{2} \sin ^{2} \vartheta_{b}+4 s_{1} s_{2} \omega_{r}^{2} \sin ^{2} \vartheta_{b}-i \Gamma m \\
& =-4 s_{1} s_{2} \sin ^{2} \vartheta_{b}\left(\omega_{\mathrm{c}}^{2}-\omega_{r}^{2}+i \frac{\Gamma m}{4 s_{1} s_{2} \sin ^{2} \vartheta_{b}}\right) \\
& \equiv-4 s_{1} s_{2} \sin ^{2} \vartheta_{b}(\chi+i a),
\end{aligned}
$$

where

$$
\chi \equiv \omega_{c}^{2}-\omega_{r}^{2}=\left(1-\frac{m^{2}}{4 \omega_{1} \omega_{2} \sin _{\vartheta_{b}}^{2}}\right) \omega_{c}^{2}
$$

describes the degrees of deviation of $E_{\mathrm{cms}}$ as determined by a pair of incident photons from the resonance energy derived from the central energy $\omega_{c}$, and $a$ is defined as

$$
a=\frac{\Gamma m \omega_{c}^{2}}{4 \omega_{1} \omega_{2} \sin ^{2} \vartheta_{b}}=\frac{1}{16 \pi}\left(\frac{g}{M}\right)^{2} \omega_{r}^{2} m^{2} .
$$

The numerator $\mathcal{N}$ in eq. (A.35) is considered to be

$$
\begin{aligned}
\mathcal{N} & =\left(\frac{g}{M}\right)^{2}\left(2 \omega_{1} \omega_{2} \sin ^{2} \vartheta_{b}\right)^{2} \\
& =16 \pi a s_{1} s_{2} \sin ^{2} \vartheta_{b}\left(\frac{\omega_{c}}{\omega_{r}}\right)^{4} \sim 16 \pi a s_{1} s_{2} \sin ^{2} \vartheta_{b},
\end{aligned}
$$

where eq. (A.37) and (A.41) are used for the second expression and the condition $\omega_{c} \sim \omega_{r}$ is required for the last step because the resonance condition $E_{\mathrm{cms}}=m$ can be satisfied dominantly with a proper choice of $\vartheta_{b}$ for a given $m$ without changing the central beam energy $\omega_{c}$ itself. Finally, the following Breit-Wigner distribution is obtained:

$$
\mathcal{M}_{s}=\frac{\mathcal{N}}{\mathcal{D}}=4 \pi \frac{a}{\chi+i a}, \quad \text { and } \quad\left|\mathcal{M}_{s}\right|^{2}=(4 \pi)^{2} \frac{a^{2}}{\chi^{2}+a^{2}} .
$$

Since we expect that $E_{\mathrm{cms}}$ is in principle uncertain due to unavoidable energy and momentum uncertainties of a selected pair of two photon wave vectors in QPS, we need to average the resonance effect over a range of $\chi$. In order to show the essence of inclusion of 
an resonance state within a range from $\chi_{-}$to $\chi_{+}$, we demonstrate the simplest averaging as follows. We define $\chi_{ \pm}$in units of $a$ as $\chi_{ \pm}= \pm \eta a$ with $\eta \gg 1$. The averaging process is expressed as

$$
\begin{aligned}
\overline{\left|\mathcal{M}_{s}\right|^{2}} & =\frac{1}{\chi_{+}-\chi_{-}} \int_{\chi_{-}}^{\chi_{+}}\left|M_{s}\right|^{2} d \chi \\
& =\frac{(4 \pi)^{2}}{2 \eta a} 2 a \tan ^{-1}(\eta)=(4 \pi)^{2} \eta^{-1} \tan ^{-1}(\eta) \\
& \approx(4 \pi)^{2} \eta^{-1} \frac{\pi}{2}=8 \pi^{2} \frac{a}{\left|\chi_{ \pm}\right|}
\end{aligned}
$$

with the approximation due to $\eta \gg 1$. Compared to non s-channel cases where $\left|\mathcal{M}_{s}\right|^{2} \propto a^{2}$, capturing a resonance within the $E_{\text {cms }}$ uncertainty has a gain of $a^{-1} \propto M^{2}$ as shown above. If the energy scale $M$ corresponds to the Planckian scale $M_{\mathrm{P}}$, this gain factor is huge even if we cannot directly capture the top of the Breit-Wigner distribution where $\left|\mathcal{M}_{s}\right|^{2} \propto(4 \pi)^{2}$ with $\chi \rightarrow 0$. This is the prominent feature of s-channel scattering including a resonance in QPS. In the following subsections, we will introduce more realistic probability distribution functions for $E_{\mathrm{cms}}$ based on the physical nature of propagating electromagnetic fields in order to implement the averaging process.

\section{A.4 Evaluation of signal yield in stimulated resonant scattering}

Let us first consider the number of scattering events in $p_{1}+p_{2} \rightarrow p_{3}+p_{4}$ with two colliding photon beams having normalized densities $\rho_{1}$ and $\rho_{2}$ with average number of photons $N_{1}$ and $N_{2}$, respectively. This is referred to as the spontaneous yield to get the signal $p_{3}$ in the final state. With the Lorentz-invariant phase space factor $d L_{\mathrm{ips}}$

$$
d L_{\mathrm{ips}}=(2 \pi)^{4} \delta\left(p_{3}+p_{4}-p_{1}-p_{2}\right) \frac{d^{3} p_{3}}{2 \omega_{3}(2 \pi)^{3}} \frac{d^{3} p_{4}}{2 \omega_{4}(2 \pi)^{3}},
$$

the spontaneous signal yield $\mathcal{Y}$ can be factorized according to the concept of time-integrated luminosity $\mathcal{L}$ times cross section $\sigma$, as follows:

$$
\begin{aligned}
\mathcal{Y} & =N_{1} N_{2}\left(\int d t d \boldsymbol{r} \rho_{1}(\boldsymbol{r}, t) \rho_{2}(\boldsymbol{r}, t) K\left(p_{1}, p_{2}\right)\right)\left(\frac{c}{2 \omega_{1} 2 \omega_{2} K\left(p_{1}, p_{2}\right)}\left|\mathcal{M}_{s}\left(p_{1}, p_{2}\right)\right|^{2} d L_{\mathrm{ips}}\right) \\
& \left.\equiv \mathcal{L}\left(p_{1}, p_{2}\right)\left[s \cdot L^{3} \cdot L^{-3} \cdot L^{-3} \cdot L / s\right)\right] \sigma\left(p_{1}, p_{2}\right)\left[L^{2}\right],
\end{aligned}
$$

where $K$ corresponds to the relative velocity of the incoming particle beams between two incident photons with velocity vectors $\boldsymbol{v}_{\mathbf{1}}$ and $\boldsymbol{v}_{\mathbf{2}}$, based on Møller's Lorentz-invariant factor [17]. The relative velocity $K$ is defined as [18]

$$
K\left(p_{1}, p_{2}\right) \equiv \sqrt{\left(\boldsymbol{v}_{\mathbf{1}}-\boldsymbol{v}_{\mathbf{2}}\right)^{2}-\frac{\left(\boldsymbol{v}_{\mathbf{1}} \times \boldsymbol{v}_{\mathbf{2}}\right)^{2}}{c^{2}}}
$$

with $c$ the velocity of light. The notation [ ] indicates units with length $L$ and time $s$. 
The concept of the cross section is convenient for fixed $p_{1}$ and $p_{2}$ beams. However, in order to implement fluctuations on the velocity vectors, which are represented by the integral on the probability density of cms-energy $W(Q)$ as a function of the combinations of energies and angles - in laboratory coordinates, denoted as

$$
Q \equiv\left\{\omega_{\alpha}, \Theta_{\alpha}, \Phi_{\alpha}\right\} \quad \text { and } \quad d Q \equiv \Pi_{\alpha} d \omega_{\alpha} d \Theta_{\alpha} d \Phi_{\alpha}
$$

for the incident beams $\alpha=1,2$ — the volume-wise interaction rate $\bar{\Sigma}$ defined below [19] ${ }^{1}$ is more straightforward than the cross section $\sigma$

$$
\begin{aligned}
\mathcal{Y} & =N_{1} N_{2}\left(\int d t d \boldsymbol{r} \rho_{1}(\boldsymbol{r}, t) \rho_{2}(\boldsymbol{r}, t)\right)\left(\int d Q W(Q) \frac{c}{2 \omega_{1} 2 \omega_{2}}\left|\mathcal{M}_{s}\left(Q^{\prime}\right)\right|^{2} d L_{\mathrm{ips}}^{\prime}\right) \\
& \equiv N_{1} N_{2} \mathcal{D}\left[s / L^{3}\right] \bar{\Sigma}\left[L^{3} / s\right]
\end{aligned}
$$

because the intermediate $K$-factor is canceled in advance of averaging over $W(Q)$, where $Q^{\prime} \equiv\left\{\omega_{\alpha}, \vartheta_{\alpha}, \phi_{\alpha}\right\}$ are kinematical parameters used for the zero- $p_{T}$ coordinate constructed from a pair of two incident waves. The conversions from $Q$ to $Q^{\prime}$ are possible through rotation functions $\vartheta_{\alpha} \equiv \mathcal{R}_{\vartheta_{\alpha}}(Q)$ and $\phi_{\alpha} \equiv \mathcal{R}_{\phi_{\alpha}}(Q)$.

We then extend the spontaneous yield to the induced yield, $\mathcal{Y}_{I}$, by adding one more beam with the central four-momentum $p_{4}$ having normalized density $\rho_{4}$ with the extended set of parameters:

$$
Q_{I} \equiv\left\{Q, \omega_{4}, \Theta_{4}, \Phi_{4}\right\} \quad \text { and } \quad d Q_{I} \equiv d Q d \omega_{4} d \Theta_{4} d \Phi_{4} .
$$

The induced yield is then expressed as

$$
\begin{aligned}
\mathcal{Y}_{I} & =N_{1} N_{2} N_{4}\left(\int d t d \boldsymbol{r} \rho_{1}(\boldsymbol{r}, t) \rho_{2}(\boldsymbol{r}, t) \rho_{4}(\boldsymbol{r}, t) V_{4}\right)\left(\int d Q_{I} W\left(Q_{I}\right) \frac{c}{2 \omega_{1} 2 \omega_{2}}\left|\mathcal{M}_{s}\left(Q^{\prime}\right)\right|^{2} d L_{\mathrm{ips}}^{\prime I}\right) \\
& \equiv N_{1} N_{2} N_{4} \mathcal{D}_{I}\left[s / L^{3}\right] \bar{\Sigma}_{I}\left[L^{3} / s\right],
\end{aligned}
$$

where $\rho_{4}(\boldsymbol{r}, t) V_{4}$ with the volume of the $p_{4}$ beam, $V_{4}$, corresponds to the probability density that describes a spacetime overlap of the $p_{1}$ and $p_{2}$ beams with the inducing beam $p_{4} ; d L_{\mathrm{ips}}^{\prime I}$ indicates the inducible phase space in which the solid angles of $p_{3}$ must be consistent so that the balancing solid angles of $p_{4}$ determined via energy-momentum conservation can be found within the distribution of the given inducing beam (in laboratory coordinates) after conversion from $p_{4}$ in the zero- $p_{T}$ coordinate system to the corresponding laboratory coordinate. $W\left(Q_{I}\right)$ is explicitly introduced as

$$
W\left(Q_{I}\right) \equiv \Pi_{\beta} G_{E}\left(\omega_{\beta}\right) G_{p}\left(\Theta_{\beta}, \Phi_{\beta}\right)
$$

with Gaussian distributions $G$ for

$$
d Q_{I} \equiv \Pi_{\beta} d \omega_{\beta} d \Theta_{\beta} d \Phi_{\beta}
$$

\footnotetext{
${ }^{1}$ See also eq. (3.80) in W. Greiner and J. Reinhardt, Quantum Electrodynamics Second Edition, Springer (1994).
} 
over $\beta=1,2,4$. The Gaussian distributions $G_{E}$ in the energy space and $G_{p}$ in the momentum space (equivalently, the polar angles in the case of photons) are introduced according to the properties of a focused coherent electromagnetic field with an axial symmetric nature for an azimuthal angle $\Phi$ around the optical axis of a focused beam, as we discuss soon.

We then specifically consider a search for signal photons $p_{3}$ for the degenerate case in the generic QPS including asymmetric collisions: $p_{c}+p_{c} \rightarrow p_{3}+p_{4}$ where $p_{1}$ and $p_{2}$ are stochastically obtained from a single focused coherent beam with central photon energy $\omega_{c}$ under the co-propagating focused coherent beam with central four-momentum $p_{4}$ for the purpose of induction. Based on the transition amplitude in eq. (A.24) and the yield expression in eq. (A.51), the induced signal yield in the degenerate case, where we combine a coherent creation beam and a coherent inducing beam with the average numbers of photons $N_{c}$ and $N_{i}$, respectively, and focus them into the common optical axis (in laboratory coordinates) is expressed as

$$
\begin{aligned}
\mathcal{Y}_{c+i}= & \left(N_{c} / 2\right)\left(N_{c} / 2\right) N_{i}\left(\int d t d \boldsymbol{r} \rho_{c}(\boldsymbol{r}, t) \rho_{c}(\boldsymbol{r}, t) \rho_{i}(\boldsymbol{r}, t) V_{i}\right) \times \\
& \left(\int d Q_{I} W\left(Q_{I}\right) \frac{c}{2 \omega_{1} 2 \omega_{2}}\left|\mathcal{M}_{s}\left(Q^{\prime}\right)\right|^{2} d L_{\mathrm{ips}}^{\prime I}\right) \\
\equiv & \frac{1}{4} N_{c}^{2} N_{i} \mathcal{D}_{I}\left[s / L^{3}\right] \bar{\Sigma}_{I}\left[L^{3} / s\right],
\end{aligned}
$$

where the factor $1 / 4$ appears for the reason explained in the paragraph just below eq. (A.24) and $\mathcal{M}_{S}$ is based on the non-degenerate case resulting in eq. (A.35). In the following, we provide detailed formulas for $\mathcal{D}_{I}$ and $\bar{\Sigma}_{I}$ in eq. (A.54).

\section{A.4.1 Properties of a Gaussian beam in vacuum}

The solution for propagation of an electromagnetic field in vacuum is known as the basic Gaussian mode [21]. In the Gaussian mode, the electric field propagating along the $z$ direction with wave number $k$ in (laboratory) spatial coordinates $(x, y, z)$ is expressed as

$$
\boldsymbol{E}(x, y, z)=\boldsymbol{E}_{0} \frac{w_{0}}{w(z)} \exp \left(-i(k z-\eta(z))-r^{2}\left(\frac{1}{w^{2}(z)}+\frac{i k}{2 R(z)}\right)\right),
$$

where the individual factors are summarized as follows.

$$
\begin{aligned}
r & =\sqrt{x^{2}+y^{2}} \\
w(z) & =w_{0} \sqrt{1+\frac{z^{2}}{z_{R}^{2}}} \\
\eta(z) & =\tan ^{-1}\left(\frac{z}{z_{R}}\right) \\
R(z) & =z\left(1+\frac{z_{R}^{2}}{z^{2}}\right) .
\end{aligned}
$$

In this, the beam waist $w_{0}$ and Rayleigh length $z_{R}$ are

$$
w_{0}=\frac{\lambda}{\pi \vartheta_{0}}, \quad z_{R}=\frac{\pi w_{0}^{2}}{\lambda}
$$


for a given wavelength $\lambda$. When a single electromagnetic field is focused with focal length $f$ and beam diameter $d$, the beam waist is related to the incident angle $\Theta_{0}$ by

$$
\Theta_{0}=\tan ^{-1}\left(\frac{d}{2 f}\right)
$$

At the focal point $z=0$, the spatial distribution of the electric field is expressed as

$$
\boldsymbol{E}(x, y, z=0)=\boldsymbol{E}_{0} \exp \left(-\frac{x^{2}+y^{2}}{w_{0}^{2}}\right) .
$$

The corresponding wave number distribution is obtained by Fourier transformation of the electric field, yielding

$$
\begin{aligned}
\hat{\boldsymbol{E}}\left(k_{x}, k_{y}, 0\right)= & \frac{1}{4 \pi^{2}} \int_{-\infty}^{\infty} \boldsymbol{E}_{0} \exp \left(-\frac{x^{2}+y^{2}}{w_{0}^{2}}\right) \times \\
& \exp \left(-i\left(k_{x} x+k_{y} y\right)\right) d x d y=\frac{w_{0}^{2} \boldsymbol{E}_{0}}{4 \pi} \exp \left(-\frac{w_{0}^{2}}{4}\left(k_{x}^{2}+k_{y}^{2}\right)\right) .
\end{aligned}
$$

The uncertainty on incident angles of wave vectors within the electric field with respect to the $z$-axis can be related to $k_{T}=\sqrt{k_{x}^{2}+k_{y}^{2}}$ via the variance

$$
\sigma_{k_{T}}^{2}=\frac{2}{w_{0}^{2}}
$$

in the Gaussian form.

For incident angles $\Theta$ defined with the transverse momenta $k_{T}$ in eq. (A.61) and the incident energies $\omega$ with $\hbar=1$,

$$
\Theta=\sin ^{-1}\left(\frac{k_{T}}{\omega}\right)
$$

and the error propagation on the incident angles is given by

$$
\begin{aligned}
\sigma_{\Theta} & =\sqrt{\left(\frac{\partial \Theta}{\partial k_{T}}\right)^{2} \sigma_{k_{T}}^{2}+\left(\frac{\partial \Theta}{\partial \omega}\right)^{2} \sigma_{\omega}^{2}} \\
& =\sqrt{\frac{1}{\langle\omega\rangle^{2}-\left\langle k_{T}\right\rangle^{2}}\left(\sigma_{k_{T}}^{2}+\left(\frac{\left\langle k_{T}\right\rangle}{\langle\omega\rangle}\right)^{2} \sigma_{\omega}^{2}\right)} \\
& =\frac{1}{\omega_{c}} \sigma_{k_{T}}=\frac{1}{\sqrt{2}} \Theta_{0},
\end{aligned}
$$

where $\langle\omega\rangle=\omega_{c},\left\langle k_{T}\right\rangle=0$, and eq. (A.61) are substituted for the last line.

The average number of photons, $N$, in a pulsed electromagnetic field can be related to the square of the electric field, $I$, by adding a Gaussian-shaped time distribution with duration $\tau$, as follows:

$$
I\left(t, x^{i}\right)=E_{0}^{2} \frac{w_{0}^{2}}{w^{2}(c t)} \exp \left(-2 \frac{x^{2}+y^{2}}{w^{2}(c t)}\right) \exp \left(-2\left(\frac{z-c t}{c \tau}\right)^{2}\right),
$$


where $E_{0}^{2}$ corresponds to $N$. The volume for the normalization is then expressed as

$$
V=\int_{-\infty}^{\infty} d x^{i} \frac{I}{E_{0}^{2}}=\left(\frac{\pi}{2}\right)^{\frac{3}{2}} w_{0}^{2} c \tau
$$

Therefore, the normalized density profile per photon, $\rho \equiv I /(N V)$, is expressed as

$$
\rho\left(t, x^{i}\right)=\frac{(2 / \pi)^{3 / 2}}{w^{2}(c t) c \tau} \exp \left(-2 \frac{x^{2}+y^{2}}{w^{2}(c t)}\right) \exp \left(-2\left(\frac{z-c t}{c \tau}\right)^{2}\right) .
$$

\section{A.4.2 Integrated inducible volume-wise interaction rate, $\bar{\Sigma}_{I}$}

With the kinematical parameters defined in a zero- $p_{T}$ coordinate as illustrated in figure 4 , $Q^{\prime} \equiv\left\{\omega_{1}, \omega_{2}, \vartheta_{1}, \vartheta_{2}, \phi_{1}, \phi_{2}\right\}$, we first discuss the integrand of the spontaneous volume-wise interaction rate in eq. (A.49) in individual zero- $p_{T}$ coordinates

$$
\Sigma^{\prime} \equiv \frac{c}{2 \omega_{1} 2 \omega_{2}}\left|\mathcal{M}_{s}\left(Q^{\prime}\right)\right|^{2} d L_{\mathrm{ips}}^{\prime}
$$

With $d^{3} p_{3}=\omega_{3}^{2} d \omega_{3} d \Omega_{3}^{\prime}$, the differential volume-wise interaction rate per solid angle $d \Omega_{3}^{\prime}$ in a zero- $p_{T}$ coordinate is expressed as

$$
\frac{d \Sigma^{\prime}}{d \Omega_{3}^{\prime}}=\frac{c}{32 \pi^{2} \omega_{1} \omega_{2}} \int_{0}^{\infty} d \omega_{3} \omega_{3} \int_{-\infty}^{\infty} d^{3} d p_{4} \frac{\left|\mathcal{M}_{s}\left(Q^{\prime}\right)\right|^{2}}{2 \omega_{4}} \delta^{4}\left(p_{3}+p_{4}-p_{1}-p_{2}\right) .
$$

We then insert the following identity

$$
1=\int_{0}^{\infty} d p_{4}^{0} \delta\left(p_{4}^{0}-\omega_{4}\right)=\int_{-\infty}^{\infty} d p_{4}^{0} 2 \omega_{4} \delta\left(p_{4}^{2}\right) \Theta\left(p_{4}^{0}\right)
$$

to derive

$$
\begin{aligned}
\int \frac{d^{3} p_{4}}{2 \omega_{4}} \delta^{4}\left(p_{3}+p_{4}-p_{1}-p_{2}\right) & =\int d^{4} p_{4} \delta\left(p_{4}^{2}\right) \Theta\left(p_{4}^{0}\right) \delta^{4}\left(p_{3}+p_{4}-p_{1}-p_{2}\right) \\
& =\delta\left(\left(p_{1}+p_{2}-p_{3}\right)^{2}\right)
\end{aligned}
$$

where $\omega_{4}>0$ is guaranteed. In an asymmetric QPS, the following relation holds due to energy-momentum conservation:

$$
\begin{aligned}
p_{4}^{2} & =\left(p_{1}+p_{2}-p_{3}\right)^{2} \\
& =2\left(\omega_{1}+\omega_{2}-\omega_{z} \cos \vartheta_{3}\right)\left(\omega_{3}-\frac{2 \omega_{1} \omega_{2} \sin ^{2} \vartheta_{b}}{\omega_{1}+\omega_{2}-\omega_{z} \cos \vartheta_{3}}\right) .
\end{aligned}
$$

Hence,

$$
\begin{aligned}
\delta\left(p_{4}^{2}\right) & =\frac{1}{2\left(\omega_{1}+\omega_{2}-\omega_{z} \cos \vartheta_{3}\right)} \delta\left(\omega_{3}-\hat{\omega}_{3}\right) \\
& =\frac{\hat{\omega}_{3}}{4 \omega_{1} \omega_{2} \sin ^{2} \vartheta_{b}} \delta\left(\omega_{3}-\hat{\omega}_{3}\right),
\end{aligned}
$$


where

$$
\hat{\omega}_{3} \equiv \frac{2 \omega_{1} \omega_{2} \sin ^{2} \vartheta_{b}}{\omega_{1}+\omega_{2}-\omega_{z} \cos \vartheta_{3}}
$$

Therefore, we can write the expression

$$
\begin{aligned}
\frac{d \Sigma^{\prime}}{d \Omega_{3}^{\prime}} & =\frac{c}{32 \pi^{2} \omega_{1} \omega_{2}} \int_{0}^{\infty} d \omega_{3} \omega_{3} \frac{\hat{\omega}_{3}}{4 \omega_{1} \omega_{2} \sin ^{2} \vartheta_{b}}\left|\mathcal{M}_{s}\left(Q^{\prime}\right)\right|^{2} \delta\left(\omega_{3}-\hat{\omega}_{3}\right) \\
& =\frac{c \hat{\omega}_{3}^{2}\left|\mathcal{M}_{s}\left(Q^{\prime}\right)\right|^{2}}{2\left(8 \pi \omega_{1} \omega_{2} \sin \vartheta_{b}\right)^{2}} .
\end{aligned}
$$

Because the incident energies and momenta fluctuate for the single creation beam, the differential volume-wise interaction rate must be averaged over possible values of $\chi$ in eq. (A.40) according to the probability distribution functions $W(Q) \equiv G_{E}\left(\omega_{1}\right) G_{E}\left(\omega_{2}\right)$ - $G_{p}\left(\Theta_{1}, \Phi_{1}\right) G_{p}\left(\Theta_{2}, \Phi_{2}\right)$ with the parameters in laboratory coordinates, where

$$
G_{E}(\omega) \equiv \frac{1}{\sqrt{2 \pi} \sigma_{\omega}} \exp \left(-\frac{(\omega-\bar{\omega})^{2}}{2 \sigma_{\omega}^{2}}\right)
$$

with mean $\bar{\omega}$ and

$$
G_{p}(\Theta, \Phi) \equiv \frac{1}{2 \pi \sigma_{\Theta}^{2}} \exp \left(-\frac{\Theta^{2}}{2 \sigma_{\Theta}^{2}}\right)=\frac{1}{\pi \Theta_{0}^{2}} \exp \left(-\frac{\Theta^{2}}{\Theta_{0}^{2}}\right)
$$

by substituting $\sigma_{\Theta}=\Theta_{0} / \sqrt{2}$ from eq. (A.63) for the second. We note that $G_{p}$ is normalized to the two-dimensional Gaussian distribution in $\Theta-\Phi$ angular space, where the $\Phi$-dependence is implicitly implemented via the axial symmetric feature of a focused beam even though the right-hand side includes only the $\Theta$-dependence. With the explicit notation $d Q \equiv d \omega_{1} d \omega_{2} d \Theta_{1} d \Theta_{2} d \Phi_{1} d \Phi_{2}$, the integrated differential volume-wise interaction rate in the zero- $p_{T}$ coordinate is then expressed as

$$
\frac{d \bar{\Sigma}^{\prime}}{d \Omega_{3}^{\prime}} \equiv \int d Q W(Q) \frac{c\left|\mathcal{M}_{s}(\mathcal{R}(Q))\right|^{2}}{2\left(8 \pi \omega_{1} \omega_{2} \sin \vartheta_{b}\right)^{2}},
$$

where $\mathcal{R}$ denotes rotation functions that convert a $Q$ given in laboratory coordinates to $Q^{\prime}$ in the corresponding zero- $p_{T}$ coordinate system.

So far, we have discussed the spontaneous scattering process resulting in the twophoton final state with $p_{3}$ and $p_{4}$. We now discuss the stimulated volume-wise interaction rate with a coherent inducing field at the spacetime where the scattering takes place. We then need to revisit the commutation relation used in eq. (A.7). To have the enhancement factor $\sqrt{N_{p_{4}}}$ appear through the second relation in eq. (A.14), both momentum and polarization states of the spontaneous $p_{4}$-wave must be identical with those in the inducing coherent field. As for the matching of polarization state, as long as we consider circular polarization states (for instance, $S=L L R R$ ), the matching is satisfied for any directions of $p_{4}$-waves in the inducing beam with the $R$-state resulting in a $p_{3}$-wave with an $R$-state. On the other hand, for the momentum state matching, we need to evaluate what fraction of the inducing beam can actually stimulate the scattering process; that is, the enhancement factor possible for the coherent state, because the focused short-pulse inducing beam has a 
spread in both momentum and energy spaces. Phase-space matching can be implemented by introducing the symbol $d L_{\mathrm{ips}}^{\prime I}$. This symbol indicates that we take into account the solid angles of signal photons, $p_{3}$, only when we can find balancing $p_{4}$ waves via energymomentum conservation within the given focused inducing field. More explicitly, we define the following relation:

$$
\bar{\Sigma}_{I} \equiv \int G_{E}\left(\omega_{4}\right) G_{p}\left(\Theta_{4}, \Phi_{4}\right) d \Omega_{4} \frac{d \Omega_{4}^{\prime}}{d \Omega_{4}} \frac{d \Omega_{3}^{\prime}}{d \Omega_{4}^{\prime}} \frac{d \bar{\Sigma}^{\prime}}{d \Omega_{3}^{\prime}},
$$

where

$$
\frac{d \Omega_{3}^{\prime}}{d \Omega_{4}^{\prime}}=\frac{d \phi_{3} \sin \vartheta_{3}}{d \phi_{4} \sin \vartheta_{4}} \frac{d \vartheta_{3}}{d \vartheta_{4}}=\frac{\sin \vartheta_{3}}{\sin \vartheta_{4}} \frac{d \vartheta_{3}}{d \vartheta_{4}}=-\left(\frac{\omega_{4}}{\omega_{3}}\right)^{2} .
$$

This is based on the energy-momentum conservation in eq. (A.27) and $d \phi_{3}=d \phi_{4}$ in zero$p_{T}$ coordinates. Because an inducible solid angle of $p_{4}$ in a zero- $p_{T}$ coordinate must match with a solid angle within the angular distribution of the inducing coherent field mapped in laboratory coordinates, $d \Omega_{4}=d \Omega_{4}^{\prime}$ must be satisfied. Therefore, the inducible volume-wise interaction rate is eventually expressed as

$$
\begin{aligned}
\bar{\Sigma}_{I}= & -\int G_{E}\left(\omega_{4}\right) G_{p}\left(\Theta_{4}, \Phi_{4}\right) d \Omega_{4}\left(\frac{\omega_{4}}{\omega_{3}}\right)^{2} \frac{d \bar{\Sigma}^{\prime}}{d \Omega_{3}^{\prime}} \\
= & \int_{0}^{2 \pi} d \Phi_{4} \int_{\pi / 2}^{0} d \Theta_{4} \sin \Theta_{4} G_{p}\left(\Theta_{4}, \Phi_{4}\right) \int d \omega_{4} G_{E}\left(\omega_{4}\right)\left(\frac{\omega_{4}}{\omega_{1}+\omega_{2}-\omega_{4}}\right)^{2} \times \\
& \int d Q W(Q) \frac{c\left|\mathcal{M}_{s}(\mathcal{R}(Q))\right|^{2}}{2\left(8 \pi \omega_{1} \omega_{2} \sin \vartheta_{b}\right)^{2}}
\end{aligned}
$$

where all integral variables are expressed by those defined in the laboratory coordinate, $Q_{I}$ as defined in eq. (A.50) that includes $Q$ in eq. (A.48).

\section{A.4.3 Spacetime overlapping factor with an inducing beam, $\mathcal{D}_{I}$}

For scattering in QPS, we introduce a common normalized density for the incident beams as $\rho_{c} \equiv \rho_{1}=\rho_{2}$ by assuming that $p_{1}$ and $p_{2}$ are stochastically selected from the single creation beam and the inducing beam $\rho_{i}$ based on eq. (A.66) as follows:

$$
\begin{aligned}
& \rho_{\mathrm{c}}\left(t, x^{i}\right)=\left(\frac{2}{\pi}\right)^{\frac{3}{2}} \frac{1}{w_{\mathrm{c}}^{2}(c t) c \tau_{\mathrm{c}}} \exp \left(-2 \frac{x^{2}+y^{2}}{w_{\mathrm{c}}^{2}(c t)}\right) \exp \left(-2\left(\frac{z-c t}{c \tau_{\mathrm{c}}}\right)^{2}\right) \\
& \rho_{\mathrm{i}}\left(t, x^{i}\right)=\left(\frac{2}{\pi}\right)^{\frac{3}{2}} \frac{1}{w_{\mathrm{i}}^{2}(c t) c \tau_{\mathrm{i}}} \exp \left(-2 \frac{x^{2}+y^{2}}{w_{\mathrm{i}}^{2}(c t)}\right) \exp \left(-2\left(\frac{z-c t}{c \tau_{\mathrm{i}}}\right)^{2}\right),
\end{aligned}
$$

where the origin is defined at $t=0$ and $x^{i}=0$ for $i=1-3$, and the pulse durations $\tau_{c}$ and $\tau_{i}$ follow independent Gaussian distributions. Because we discussed the angular spread only at the origin in eq. (A.60), where the maximum interaction rate is expected, we limit the region of interest to within the Rayleigh length $z_{R}$ in order to provide a conservative 
estimate of the yield. Therefore, with $V_{i}=(\pi / 2)^{3 / 2} w_{i 0}^{2} c \tau_{i}$ from eq. (A.65), the overlap factor is approximated as

$$
\begin{aligned}
\mathcal{D}_{I} & \approx \int_{-z_{R} / c}^{0} d t \int_{-\infty}^{\infty} d x^{i} \rho_{\mathrm{c}}^{2}\left(t, x^{i}\right) \rho_{\mathrm{i}}\left(t, x^{i}\right) V_{\mathrm{i}} \\
& =\left(\frac{2}{\pi}\right)^{\frac{3}{2}} \frac{1}{c} \frac{\tau_{\mathrm{i}}}{\tau_{\mathrm{c}}} \frac{1}{\sqrt{\tau_{\mathrm{c}}^{2}+2 \tau_{\mathrm{i}}^{2}}} w_{\mathrm{i} 0}^{2} \int_{-z_{R} / c}^{0} d t \frac{1}{w_{\mathrm{c}}^{4}(c t)+2 w_{\mathrm{c}}^{2}(c t) w_{\mathrm{i}}^{2}(c t)},
\end{aligned}
$$

where the integrand

$$
\frac{A}{w_{\mathrm{c}}^{2}(c t)}-\frac{B}{w_{\mathrm{c}}^{2}(c t)+2 w_{\mathrm{i}}^{2}(c t)}
$$

with

$$
A=\frac{1}{2 w_{\mathrm{i} 0}^{2}\left\{1-\left(\frac{z_{\mathrm{c} R}}{z_{\mathrm{i} R}}\right)^{2}\right\}}
$$

and

$$
B=\frac{1}{1-\left(\frac{z_{\mathrm{c} R}}{z_{\mathrm{i} R}}\right)^{2}}\left\{\frac{1}{2 w_{\mathrm{i} 0}^{2}}+\frac{1}{w_{\mathrm{c} 0}^{2}}\left(\frac{z_{\mathrm{c} R}}{z_{\mathrm{i} R}}\right)^{2}\right\} .
$$

Finally, the spacetime overlap factor for the two effective beams in QPS is expressed as

$$
\mathcal{D}_{I} \approx \sqrt{\frac{2}{\pi}} \frac{1}{c^{2}} \frac{\tau_{\mathrm{i}}}{\tau_{\mathrm{c}}} \frac{1}{\sqrt{\tau_{\mathrm{c}}^{2}+2 \tau_{\mathrm{i}}^{2}}} \frac{\lambda_{\mathrm{i}}^{2}}{\lambda_{\mathrm{i}}^{2}-\lambda_{\mathrm{c}}^{2}}\left[\frac{1}{\lambda_{\mathrm{c}}} \tan ^{-1}\left(\frac{\lambda}{\lambda_{\mathrm{c}}}\right)-\frac{1}{\lambda_{\mathrm{ci}}} \tan ^{-1}\left(\frac{\lambda}{\lambda_{\mathrm{ci}}}\right)\right]
$$

with $\lambda_{\mathrm{ci}} \equiv \sqrt{\frac{\lambda_{\mathrm{c}}^{2}+2 \lambda_{\mathrm{i}}^{2}}{3}}$.

Open Access. This article is distributed under the terms of the Creative Commons Attribution License (CC-BY 4.0), which permits any use, distribution and reproduction in any medium, provided the original author(s) and source are credited.

\section{References}

[1] Particle Data Group, Review of Particle Physics, Phys. Rev. D 98 (2018) 030001 [INSPIRE].

[2] Y. Nambu, Axial vector current conservation in weak interactions, Phys. Rev. Lett. 4 (1960) 380 [INSPIRE].

[3] J. Goldstone, Field Theories with Superconductor Solutions, Nuovo Cim. 19 (1961) 154 [INSPIRE].

[4] A. Arvanitaki, S. Dimopoulos and K. Van Tilburg, Sound of Dark Matter: Searching for Light Scalars with Resonant-Mass Detectors, Phys. Rev. Lett. 116 (2016) 031102 [arXiv: 1508.01798] [INSPIRE].

[5] Y. Fujii and K. Homma, An approach toward the laboratory search for the scalar field as a candidate of Dark Energy, Prog. Theor. Phys. 126 (2011) 531 [Erratum ibid. 2014 (2014) 089203] [arXiv: 1006.1762] [INSPIRE]. 
[6] K. Homma, T. Hasebe and K. Kume, The first search for sub-eV scalar fields via four-wave mixing at a quasi-parallel laser collider, Prog. Theor. Exp. Phys. 2014 (2014) 083C01 [arXiv: 1405.4133] [INSPIRE].

[7] T. Hasebe et al., Search for sub-eV scalar and pseudoscalar resonances via four-wave mixing with a laser collider, Prog. Theor. Exp. Phys. 2015 (2015) 073C01 [arXiv:1506.05581] [INSPIRE].

[8] A. Nobuhiro et al., Extended search for sub-eV axion-like resonances via four-wave mixing with a quasi-parallel laser collider in a high-quality vacuum system, Prog. Theor. Exp. Phys. 2020 (2020) 073C01 [arXiv: 2004.10637] [INSPIRE].

[9] K. Homma and Y. Toyota, Exploring pseudo-Nambu-Goldstone bosons by stimulated photon colliders in the mass range $0.1 \mathrm{eV}$ to $10 \mathrm{keV}$, Prog. Theor. Exp. Phys. 2017 (2017) 063C01 [arXiv: 1701.04282] [INSPIRE].

[10] Canon, Pulsed klystron E3712, (2020)

https://etd.canon/ja/product/category/microwave/klystron.html.

[11] Y.-F. Chen et al., Microwave photon counter based on Josephson junctions, Phys. Rev. Lett. 107 (2011) 217401.

[12] K. Inomata, Single microwave-photon detector using an artificial $\Lambda$-type three-level system, Nature Commun. 7 (2016) 12303 [arXiv:1601.05513].

[13] K. Homma, K. Matsuura and K. Nakajima, Testing helicity-dependent $\gamma \gamma \rightarrow \gamma \gamma$ scattering in the region of MeV, Prog. Theor. Exp. Phys. 2016 (2016) 013C01 [arXiv:1505.03630] [INSPIRE].

[14] P. Wang, H. Chen, J. Shi, I. Syratchev, W. Wuensch and H. Zha, The RF Design of a Compact, High Power Pulse Compressor with a Flat Output Pulse, in proceedings of the 7th International Particle Accelerator Conference (IPAC 2016), Busan, Republic of Korea, 8-13 May 2016 [INSPIRE] and online pdf version at http://accelconf.web.cern.ch/accelconf/ipac2016/papers/thpmw022.pdf.

[15] S.V. Samsonov et al., Compression of frequency-modulated pulses using helically corrugated waveguides and its potential for generating multigigawatt rf radiation, Phys. Rev. Lett. 92 (2004) 118301.

[16] E. Brookner, Phased-Array Radars, Sci. Am. 252 (1985) 94.

[17] C. Møller, General Properties of the Characteristic Matrix in the Theory of Elementary Particles. I, 1st edition, I kommission hos Ejnar Munksgaard, Copenhagen Denmark (1946).

[18] M.A. Furman, The Møller Luminosity Factor, LBNL-53553 [CBP Note-543] [https://doi.org/10.2172/836235].

[19] J.D. Bjorken and S.D. Drell, Relativistic Quantum Mechanicsh, McGraw-Hill, Inc. (1964).

[20] R.J. Glauber, Coherent and incoherent states of the radiation field, Phys. Rev. 131 (1963) 2766 [INSPIRE].

[21] A. Yariv, Optical Electronics in Modern Communications, Oxford University Press (1997). 